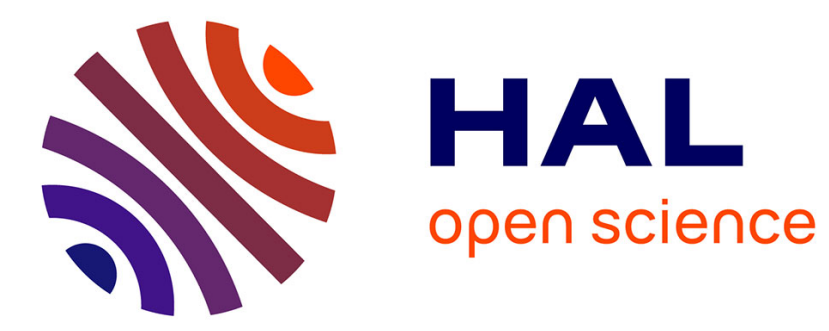

\title{
The no-response approach and its relation to non-iterative methods for the inverse scattering
}

Naofumi Honda, Gen Nakamura, Roland Potthast, Mourad Sini

\section{To cite this version:}

Naofumi Honda, Gen Nakamura, Roland Potthast, Mourad Sini. The no-response approach and its relation to non-iterative methods for the inverse scattering. 2007. hal-00136019

\section{HAL Id: hal-00136019 \\ https://hal.science/hal-00136019}

Preprint submitted on 12 Mar 2007

HAL is a multi-disciplinary open access archive for the deposit and dissemination of scientific research documents, whether they are published or not. The documents may come from teaching and research institutions in France or abroad, or from public or private research centers.
L'archive ouverte pluridisciplinaire HAL, est destinée au dépôt et à la diffusion de documents scientifiques de niveau recherche, publiés ou non, émanant des établissements d'enseignement et de recherche français ou étrangers, des laboratoires publics ou privés. 


\title{
The no-response approach and its relation to non-iterative methods for the inverse scattering
}

\author{
Naofumi Honda • Gen Nakamura • \\ Roland Potthast • Mourad Sini
}

Received: 12 August 2005 / Revised: 8 March 2006

(C) Springer-Verlag 2006

\begin{abstract}
This paper addresses the inverse obstacle scattering problem. In the recent years several non-iterative methods have been proposed to reconstruct obstacles (penetrable or impenetrable) from near or far field measurements. In the chronological order, we cite among others the linear sampling method, the factorization method, the probe method and the singular sources method. These methods use differently the measurements to detect the unknown obstacle and they require the use of many incident fields (i.e. the full or a part of the far field map). More recently, two other approaches have been added. They are the no-response test and the range test. Both of them use few incident fields to detect some informations about the scatterer. All the mentioned methods are based on building functions depending on some parameter. These functions share the property that their behaviors with respect to the parameter change drastically. The surface of the obstacle is located at most in the interface where these functions become large. The goal of this work is to investigate the relation between some of the non-iterative reconstruction schemes regarding the convergence issue. A given method is said to be convergent if it reconstructs a part or the entire obstacle by using few or many incident fields respectively. For simplicity we consider the obstacle reconstruction problem from far field data for the Helmholtz equation.
\end{abstract}

Gen Nakamura is partially supported by Grant-in-Aid for Scientific research (B)(2)(N.14340038) of Japan Society for Promotion of Science.

Mourad Sini is supported by Japan Society for Promotion of Science.

N. Honda

Department of Mathematics, Hokkaido University, Sapporo 060-0810, Japan

G. Nakamura

Department of Mathematics, Hokkaido University, Sapporo 060-0810, Japan

R. Potthast

Institute for Numerical and Applied Mathematics, University of Gottingen-Tomo-science GbR,

Wolfsburg-Gottingen, Germany

M. Sini (凶)

Department of Mathematics, Hokkaido University, Sapporo 060-0810, Japan

e-mail:sini@math.sci.hokudai.ac.jp 


\section{Introduction}

Let $D$ be a bounded domain in $\mathbb{R}^{3}$ with $C^{2}$ boundary $\partial D$. We consider the acoustic inverse scattering problem. The propagation of time-harmonic acoustic fields in a homogeneous media is governed by the Helmholtz equation

$$
\Delta u+\kappa^{2} u=0 \quad \text { in } \mathbb{R}^{3} \backslash \bar{D}
$$

where $\kappa$ is the real positive wave number. At the boundary of sound-soft scatterers the total field $u$ satisfies the Dirichlet boundary condition

$$
u=0 \quad \text { on } \partial D .
$$

Given an incident field $u^{i}$ which satisfies $\Delta u^{i}+\kappa^{2} u^{i}=0$ in $\mathbb{R}^{3}$ we look for solutions $u:=u^{i}+u^{s}$ of (1.1) and (1.2) where the scattered field $u^{s}$ is assumed to satisfy the Sommerfeld radiation condition

$$
\lim _{r \rightarrow \infty} r\left(\frac{\partial u^{s}}{\partial r}-i \kappa u^{s}\right)=0
$$

$r=|x|$ and the limit is uniform with respect to all the directions $\theta:=\frac{x}{|x|}$. It is well known (see [6] or [18]) that this reflected field satisfies the following asymptotic property,

$$
u^{s}(x)=\frac{\mathrm{e}^{i \kappa r}}{r} u^{\infty}\left(\theta, u^{i}\right)+O\left(r^{-2}\right), \quad r \rightarrow \infty,
$$

where the function $u^{\infty}\left(\cdot, u^{i}\right)$ defined on the unit sphere $\mathbb{S}$ is called the far-field associated to the incident field $u^{i}$. Taking particular incident fields given by the plane waves, $u^{i}(x, d):=\mathrm{e}^{i \kappa d \cdot x}, d \in \mathbb{S}$, we define the far-field pattern $u^{\infty}(\theta, d)$ for $(\theta, d) \in \mathbb{S} \times \mathbb{S}$. Note that we changed in the notation of $u^{\infty}$ the argument $u^{i}$ by the direction of incidence $d$. We will keep this notation for the rest of the paper. Analogously, for an incident point source $\Phi(\cdot, z)$, where

$$
\Phi(x, y):=\frac{1}{4 \pi} \frac{\mathrm{e}^{i \kappa|x-y|}}{|x-y|}, \quad x \neq y, x, y \in \mathbb{R}^{3} .
$$

is the fundamental solution of $\Delta+\kappa^{2}$ on $\mathbb{R}^{3}$, we denote the scattered field by $\Phi^{s}(\cdot, z)$ and its far field pattern by $\Phi^{\infty}(\cdot, z)$. The problem we are concerned with is the following

Shape reconstruction problem Given $u^{\infty}(\cdot, \cdot)$ on $\mathbb{S} \times \mathbb{S}$ for the scattering problem (1.1), (1.2), (1.3) find the obstacle $D$.

This problem has been well studied, see [6] or [18] for more details. Several methods have been created to solve this problem. Often, the methods are classified into the categories of iterative methods and non-iterative methods. We are concerned with the non-iterative methods as linear sampling method [5], factorization method [11], probe method [7], singular sources method [18], no-response test [13] and range test [19]. All these methods make different use of the given data. The four first methods require the far field map (or its restriction to some part of $\mathbb{S}$ ) and reconstruct the entire obstacle. The two last methods, as proposed in [13] and [19], use few incident fields and detect some informations about the unknown scatterer. In this sense, a method using many incident fields or waves (i.e. the full or part of the far field map) is called a multi-wave 
method while if it is using few incident waves it is called a one-wave method. Our study is motivated by the following facts. All the mentioned methods are based on the use of some functions which depend on some parameter. These functions share the property that their behaviors change drastically with respect to the parameter and they split the ambient space, $\mathbb{R}^{3}$, into two separated parts. The believe is that the surface of the unknown obstacle is located in the interface of these two parts. These functions are called indicator functions and a given method is said to be convergent if this believe is justified.

The goal of this paper is to clarify the relation between the mentioned methods by giving some links between the corresponding indicator functions. In Sect. 2, we give the first version of the no-response test, which is the multi-wave version of the one-wave method given in [13], and justify its convergence.

To deal with this inverse scattering problem via the probe method, in [8] the author proceeds in two steps. The first step is to compute the near field from the far field and the second step is to detect the obstacle from this near field. The near field is given by the Dirichlet to Neumann map of the boundary problem stated on some artificially introduced domain $\Omega$ containing the unknown obstacle. In this paper, we state the natural far field version of the probe method. This version uses directly the far field (in one step) to detect the obstacle. We show also that the indicator of this far field version and the (original) one of near field version are equivalent regarding the blow up property.

The singular sources method computes the scattered field $\Phi^{S}(\cdot, z)$ of the incident point sources $\Phi(\cdot, z)$ from the far field where $z$ is outside the obstacle $D$. We reformulate the singular sources method in a way which enables us to compute its indicator function for any point $z$ inside or outside $D$. For $z$ outside $\bar{D}$, it coincides with the original one, i.e. $\Phi^{S}(z, z)$.

We find out that the indicator function of the far field version of probe method and the one of the reformulated singular sources method coincide. The obstacle $D$ should be characterized by the set of points $z$ for which this indicator function blows-up. The behavior of this indicator function with respect to the parameter $z$ is similar to the one of the linear sampling method or the factorization method [11]. But it has an opposite behavior in the sense that it is bounded outside the obstacle $D$ and becomes large when approaching $D$ and stay unbounded inside $D$. This is the object of Sect. 3 .

In Sect. 4, we introduce a second version of the no-response test for reconstructing the obstacle $D$ from the knowledge of the far-field pattern. We base this second version on a combination of the superposition principle with the range test idea given in [19]. This second version of the no-response test can also be seen as a multiwave version of the range test which is different from the multiwave range test as described in [20]. We give the justification of its convergence.

In Sect. 5, we recall the linear sampling method, see [5] and [4], and show how it is related to the two proposed methods by explaining how the convergence of the linear sampling method implies the convergence of the second version of the no-response test. That is, we will show that the singular sequence (for its definition, see the caption of Fig. 1) creating the blow-up for the linear sampling method can be used to create the blow-up for the no-response test.

Finally, in Sect. 6, we show that the two versions of the no-response test are equivalent with respect to their convergence. Precisely, we give a link between the singular sequences creating the blowup property for the indicator functions of both the two 


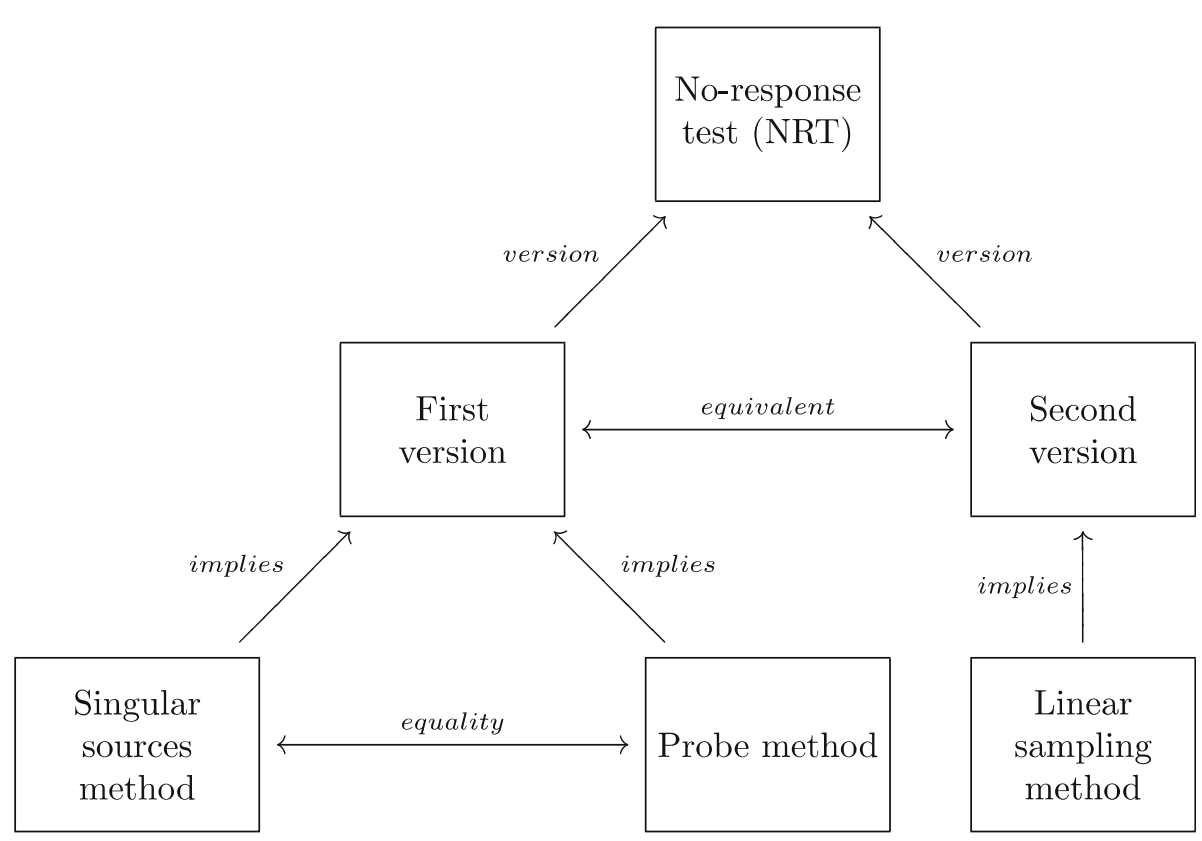

Fig. 1 The Diagram shows the relation between the two versions of the no-response test and several non-iterative methods for the inverse scattering theory. All these methods are based on indicator functions which depend on a parameter. The convergence of these methods is interpreted by the existence of sequences of functions which approximate a singular solution of the Helmholtz equation and create the blowup property for the corresponding indicator functions. We call these sequences singular sequences. In this sense, the words "implies" and "equivalent" mean that knowing the singular sequence for one method, we can know the corresponding singular sequence for the other method, and viceversa. The word "equality" means that effectively the two methods have equal indicator functions. Finally, the word "version" means that, based on the no-response idea, we build two methods using two different indicator functions

methods. Altogether, the relations between the different methods are graphically displayed in Fig. 1.

In the next sections, we make use of the following definition.

Definition 1.1 We call a bounded domain $B$, with $C^{2}$ regular boundary, such that $\mathbb{R}^{3} \backslash \bar{B}$ is connected a non-vibrating domain if $\kappa^{2}$ is not a Dirichlet eigenvalue for $(-\Delta)$. If this last condition is not satisfied, we say $B$ is vibrating.

It is well known that if $\kappa>0$ and $u \in C^{2}\left(\mathbb{R}^{3} \backslash \bar{B}\right)$ satisfies $(\Delta+\kappa) u=0$ in $\mathbb{R}^{3} \backslash \bar{B}$, $u=0$ on $\partial B$ and the Sommerfeld radiation conditions (1.3) then $u=0$ in $\mathbb{R}^{3} \backslash \bar{B}$, see for instance [6]. It is also worth noticing that the set of vibrating domains included in given bounded domain $\Omega$ is "negligible". This is due to the facts that (1) for any fixed $B$, the sequence of eigenvalues is tending to infinity and (2) by increasing strictly $B$ every eigenvalue strictly decreases. A justification of this second property can be found in ([22], page 29). We also want to mention that we can always test whether any given domain is non-vibrating by using the Courant min-max principle.

Some of these results have been announced in [15]. 


\section{The first version of the no-response test for the scattering problem}

The goal of this section is to develop a multi-wave formulation for the no response test (NRT) for the acoustic scattering problem. We will introduce the basic idea of the NRT and then prove its convergence. We start with some preparations. We set $\mathbb{S}$ to be the unit sphere in $\mathbb{R}^{3}$.

Let $g \in L^{2}(\mathbb{S})$. It is well known (see [6] and [18]) that the scattered field associated with the Herglotz incident field $v_{g}^{i}:=v_{g}$ defined by

$$
v_{g}(x):=\int_{\mathbb{S}} \mathrm{e}^{i \kappa x \cdot d} g(d) \mathrm{d} s(d), \quad x \in \mathbb{R}^{3},
$$

is given by

$$
v_{g}^{s}(x):=\int_{\mathbb{S}} u^{s}(x, d) g(d) \mathrm{d} s(d), \quad x \in \mathbb{R}^{3} \backslash D,
$$

and its far field is given by

$$
v_{g}^{\infty}(\theta):=\int_{\mathbb{S}} u^{\infty}(\theta, d) g(d) \mathrm{d} s(d), \quad \theta \in \mathbb{S}
$$

We base the method on the representation

$$
u^{\infty}(\theta, d)=\frac{1}{4 \pi} \int_{\partial D}\left\{\frac{\partial u^{s}(y, d)}{\partial v} \mathrm{e}^{-i \kappa \theta \cdot y}-\frac{\partial e^{-i \kappa \theta \cdot y}}{\partial v} u^{s}(y, d)\right\} \mathrm{d} s(y)
$$

given by using the Green's formula in $\mathbb{R}^{3} \backslash \bar{D}$ for $u^{s}(\cdot, d)$ and $\Phi(\cdot, y)$ and their asymptotic behavior at infinity (see [6], Theorem 2.5) where the normal is directed into inside $D$. The representation of the scattered field $\Phi^{s}(x, z)$ for $x, z \in \mathbb{R}^{3} \backslash \bar{D}$ is given by Green's formula

$$
\Phi^{S}(x, z)=\int_{\partial D}\left\{\frac{\partial \Phi^{s}(y, z)}{\partial v(y)} \Phi(x, y)-\Phi^{S}(y, z) \frac{\partial \Phi(x, y)}{\partial v(y)}\right\} \mathrm{d} s(y), \quad x, z \in \mathbb{R}^{3} \backslash \bar{D}
$$

Using

$$
0=\int_{\partial D}\left\{\frac{\partial \Phi(y, z)}{\partial v(y)} \Phi(x, y)-\Phi(y, z) \frac{\partial \Phi(x, y)}{\partial v(y)}\right\} \mathrm{d} s(y), \quad x, z \in \mathbb{R}^{3} \backslash \bar{D}
$$

this can be transformed into

$$
\Phi^{s}(x, z)=\int_{\partial D} \frac{\partial\left(\Phi^{s}+\Phi\right)(y, z)}{\partial v(y)} \cdot \Phi(x, y) \mathrm{d} s(y), \quad x, z \in \mathbb{R}^{3} \backslash \bar{D} .
$$


Now, consider a couple of densities $(f, g) \in L^{2}(\mathbb{S}) \times L^{2}(\mathbb{S})$. We replace $\theta$ by $-\theta$ in Eq. (2.8), multiply the result by $f(\theta) g(d)$ and integrate on $\mathbb{S} \times \mathbb{S}$ to calculate

$$
\begin{aligned}
& \int_{\mathbb{S}} \int_{\mathbb{S}} u^{\infty}(-\theta, d) f(\theta) g(d) \mathrm{d} s(\theta) \mathrm{d} s(d) \\
&= \frac{1}{4 \pi} \int_{\partial D}\left\{\int_{\mathbb{S}} \frac{\partial u^{s}(y, d)}{\partial v} g(d) \mathrm{d} s(d) \cdot \int_{\mathbb{S}} \mathrm{e}^{i \kappa \theta \cdot y} f(\theta) \mathrm{d} s(\theta)\right. \\
&-\int_{\mathbb{S}} \frac{\partial \mathrm{e}^{i \kappa \theta \cdot y}}{\partial v} f(\theta) \mathrm{d} s(\theta) \cdot \int_{\mathbb{S}} \underbrace{u^{s}(y, d)}_{=-u^{i}(y, d)} g(d) \mathrm{d} s(d)\} \mathrm{d} s(y) \\
&= \frac{1}{4 \pi} \int_{\partial D}\left\{\frac{\partial v_{g}^{i}}{\partial v}(y) v_{f}^{i}(y)+\frac{\partial v_{f}}{\partial v}(y) v_{g}^{i}(y)\right\} \mathrm{d} s(y) .
\end{aligned}
$$

Now, we state the definition of the first version of the no-response test.

Definition 2.1 (The first version of the no response method) Let $B$ be any nonvibrating domain. We define the indicator function for the multi-wave no-response test by

$$
I_{1}(B):=\lim _{\epsilon \rightarrow 0} \sup \left\{I_{1, \epsilon}(f, g):\left\|v_{f}\right\|_{L^{2}(\partial B)}<\epsilon,\left\|v_{g}\right\|_{L^{2}(\partial B)}<\epsilon\right\}
$$

with

$$
I_{1, \epsilon}(f, g):=\left|\int_{\mathbb{S}} \int_{\mathbb{S}} u^{\infty}(-\theta, d) f(\theta) g(d) d s(\theta) d s(d)\right| .
$$

For the set $\mathcal{G}$ of non-vabriting domains $B$ no response test calculates the indicator function $I_{1}(B)$ and builds the intersection

$$
D_{\mathrm{rec}, 1}:=\bigcap_{B \in \boldsymbol{B}_{1}} B
$$

where

$$
\boldsymbol{B}_{1}:=\left\{B \in \mathcal{G}: I_{1}(B)=0\right\} .
$$

After the above preparations we can prove the following characterization of $D$ from the far field pattern, which provides a convergence result for the no response test for reconstructing the inclusion $D$.

Theorem 2.2 (Convergence of the first version) Let $\mathcal{G}$ as in Definition 2.1. We have

1. If $\bar{D} \subset B$ then $I_{1}(B)=0$.

2. If $\bar{D} \not \subset B$ then $I_{1}(B)=\infty$.

Thus the unknown scatterer is given by the intersection of all test domains $B$ for which $I_{1}(B)$ is zero, i.e

$$
\bar{D}=D_{\text {rec }, 1}
$$


Proof First, consider the case where $\bar{D} \subset B$. For $\left\|v_{g}\right\|_{L^{2}(\partial B)}<\epsilon$ then from the regularity theory of the very weak solutions of the elliptic problems, see [16], we have $\left\|v_{g}\right\|_{L^{2}(B)}<c \epsilon$. Hence by interior estimates we have $\left\|v_{g}\right\|_{C^{1}(\partial D)}<c^{\prime} \epsilon$ and then

$$
\left\|v_{g}^{s}\right\|_{C(\partial D)}<c_{1} \epsilon, \quad\left\|\frac{\partial v_{g}^{s}}{\partial v}\right\|_{C(\partial D)}<c_{2} \epsilon
$$

with some constants $c, c^{\prime}, c_{1}$ and $c_{2}$. Using (2.12) and the fact that $\left\|v_{f}\right\|_{C^{1}(\bar{D})}<\tilde{c} \epsilon$, we obtain

$$
\left|I_{1, \epsilon}(f, g)\right| \leq C \epsilon^{2}
$$

with some constant $C$ and thus

$$
I_{1}(B)=\lim _{\epsilon \rightarrow 0} \sup \left\{I_{1, \epsilon}(f, g):\left\|v_{g}\right\|_{L^{2}(\partial B)}<\epsilon,\left\|v_{f}\right\|_{L^{2}(\partial B)}<\epsilon\right\}=0 .
$$

Second, $\bar{D} \not \subset B$. Let $z \in \partial D$ such that $z$ is on the boundary of the unbounded component of $\mathbb{R}^{3} \backslash \overline{D \cup B}$. Then, there exists a sequence of points

$$
\left(z_{p}\right)_{p \in \mathbb{N}} \subset \mathbb{R}^{3} \backslash(\overline{B \cup D})
$$

such that $z_{p}$ tends to $z$. We consider the sequence of point sources $\Phi\left(\cdot, z_{p}\right)$. We set $B_{p}$ as a sequence of non-vibrating domains such that $\overline{B \cup D} \subset B_{p}$ and $z_{p} \in \mathbb{R}^{n} \backslash \overline{B_{p}}$. In this case, due to the denseness property of the Herglotz wave operator (see [18], Lemma 3.1.3) we take $g_{n}^{p}$ as a sequence such that for every $p$ fixed

$$
\left\|v_{g_{n}^{p}}-\frac{\epsilon}{2} \alpha_{p} \Phi\left(\cdot, z_{p}\right)\right\|_{L^{2}\left(\partial B_{p}\right)} \rightarrow 0, \quad n \rightarrow \infty
$$

where

$$
\alpha_{p}:=\left\|\Phi\left(\cdot, z_{p}\right)\right\|_{L^{2}(\partial B)}^{-1} .
$$

Hence, by a combination of (2.21) and (2.22) and the well-posedness of the interior Dirichlet problem in $B_{p}$ we derive that for every $p$ fixed we have

$$
\left\|v_{g_{n}^{p}}\right\|_{L^{2}(\partial B)}<\epsilon
$$

for $n$ large enough. On the other hand, from (2.12), replacing $(f, g)$ by $\left(g_{n}^{p}, g_{n}^{p}\right)$, we deduce that

$$
\begin{aligned}
& \lim _{n \rightarrow \infty} \int_{\mathbb{S}} \int_{\mathbb{S}} u^{\infty}(-\theta, d) g_{n}^{p}(\theta) g_{n}^{p}(d) \mathrm{d} s(\theta) \mathrm{d} s(d) \\
& =\frac{\epsilon^{2} \alpha_{p}^{2}}{16 \pi} \int_{\partial D} \frac{\partial\left(\Phi^{s}+\Phi\right)\left(y, z_{p}\right)}{\partial v(y)} \cdot \Phi\left(y, z_{p}\right) \mathrm{d} s(y) \\
& =\frac{\epsilon^{2} \alpha_{p}^{2}}{16 \pi} \Phi^{s}\left(z_{p}, z_{p}\right) .
\end{aligned}
$$

Hence using the property

$$
\left|\Phi^{S}\left(z_{p}, z_{p}\right)\right| \geq c_{1}\left[d\left(z_{p}, \partial D\right)\right]^{-1}
$$

as shown in Theorem 2.1.15 of [18] and the fact that

$$
\alpha_{p}^{2}:=\left\|\Phi\left(\cdot, z_{p}\right)\right\|_{L^{2}(\partial B)}^{-2} \geq c_{2}\left[\ln \left(d\left(z_{p}, \partial B\right)\right)\right]^{-1}
$$


for some positive constants $c_{1}, c_{2}$, we deduce that

$$
\lim _{p \rightarrow \infty} \lim _{n \rightarrow \infty} \int_{\mathbb{S}} \int_{\mathbb{S}} u^{\infty}(-\theta, d) g_{n}^{p}(\theta) g_{n}^{p}(d) \mathrm{d} \theta \mathrm{d} s(d)=\infty .
$$

Then $I_{1}(B)=\infty$.

From the points 1 and $2, I_{1}$ may have only the values 0 and $+\infty$. In addition, $I_{1}$, as set function, is monotonically decreasing. From such properties, we get $\bar{D}=D_{\text {rec }, 1}$.

\section{The probe and singular sources methods for far field data coincide}

\subsection{Singular sources method}

The idea of the singular sources method is firstly to compute $\Phi^{s}(z, z)$ from the far field and secondly to evaluate its behavior with respect to the parameter $z$. The observation is that when $z$ approaches $\partial D$ then $\Phi^{s}(z, z)$ blows-up. To compute $\Phi^{s}(z, z)$ from the far field pattern, in [18], the author uses the so-called back-projection operator, see ([18], Definition 3.1.5 and Theorem 3.1.6). Its derivation is based on a mixed reciprocity relation. However, the calculation of $\Phi^{S}(z, z)$ can be justified directly by the use of the identity (2.8) and arguing as in (2.21)-(2.25). Indeed, let the sequence $\left(g_{n}^{z}\right)_{n \in \mathbb{N}} \subset L^{2}(\mathbb{S})$ be constructed as we did for $\left(g_{n}^{p}\right)_{n \in \mathbb{N}}$ in the proof of Theorem 2.2, where the point $z$ plays the role of the point $z_{p}$, with the slight difference in (2.21) where we replace $\frac{\epsilon}{2} \alpha_{p} \Phi\left(\cdot, z_{p}\right)$ by $\Phi(\cdot, z)$.

We obtain the following proposition

\section{Proposition 3.1}

$$
\Phi^{S}(z, z)=4 \pi \lim _{n \rightarrow \infty} \int_{\mathbb{S}} \int_{\mathbb{S}} u^{\infty}(-\theta, d) g_{n}^{z}(\theta) g_{n}^{z}(d) \mathrm{d} s(\theta) \mathrm{d} s(d) .
$$

Note that here we obtain an approximation with the quadratic form, whereas in [18], Theorem 3.1.6, one needs the full bilinear form

$$
\int_{\mathbb{S}} \int_{\mathbb{S}} u^{\infty}(-\theta, d) g(\theta) f(d) \mathrm{d} s(\theta) \mathrm{d} s(d)
$$

with $f, g \in L^{2}(\mathbb{S})$ chosen appropriately to obtain the approximation of $\Phi^{s}(z, z)$. Thus, the proof via equation (2.8) yields better results than the application of the mixed reciprocity relation. But the computation of $\Phi^{s}(z, z)$ is meaningful only if $z \in \mathbb{R}^{3} \backslash \bar{D}$. To state the singular sources method for any point $z \in \mathbb{R}^{3}$, we reformulate it in the following way:

Definition 3.2 (The indicator function) Let $\Omega$ be a large but bounded domain containing the unknown obstacle $D$. Let $z \in \Omega$. We denote $\mathcal{C}_{z}$ to be the set of continuous curves $c_{z}$ which join the point $z$ to the boundary $\partial \Omega$. For any curve $c_{z} \in \mathcal{C}_{z}$, we define $\Omega_{z}$ to be a $C^{2}$-regular domain contained strictly in $\Omega \backslash c_{z}$.

Let $v_{g_{n}^{z}}$ be a sequence of Herglotz waves which approximate $\Phi(\cdot, z)$ and set

$$
I_{s s m}\left(z, c_{z}, \Omega_{z}\right):=4 \pi \lim _{n \rightarrow \infty} \int_{\mathbb{S}} \int_{\mathbb{S}} u^{\infty}(-\theta, d) g_{n}^{z}(\theta) g_{n}^{z}(d) \mathrm{d} s(\theta) \mathrm{d} s(d) .
$$


Then, we define the indicator function $I(z)$ by

$$
I(z):=\inf _{\left\{c_{z} \in \mathcal{C}_{z}\right\}} \sup _{\left\{\Omega_{z} \subset\left(\Omega \backslash c_{z}\right)\right\}}\left\{\left|I_{s s m}\left(z, c_{z}, \Omega_{z}\right)\right|\right\}
$$

Remark 3.3 In the last definition if $\Omega_{z}$ is a vibrating domain, then we replace it with a larger non-vibrating domain $\tilde{\Omega}_{z}$ such that $z \notin \tilde{\Omega}_{z}$, then we approximate $\Phi(\cdot, z)$ on $\tilde{\Omega}_{z}$ and then we get also the same approximation on $\Omega_{z}$. This means that we can define the functional $I_{s s m}\left(z, c_{z}, \Omega_{z}\right)$ for any $C^{2}-$ regular domains.

We state the following conjecture as a claim. Its full proof is not achieved yet. In Sect. 3.3, we give some comments on its justification.

Claim 3.4 Let $z \in \Omega$ and $c_{z} \in \mathcal{C}_{z}$.

1. If the domain $\Omega_{z}$ is such that $\Omega_{z} \subset \overline{\Omega_{z}} \subset \Omega \backslash c_{z}$ and $\bar{D} \subset \Omega \backslash c_{z}$ then we have:

$$
I_{s s m}\left(z, c_{z}, \Omega_{z}\right)=\Phi^{s}(z, z) .
$$

2. If the domain $\Omega_{z}$ is such that $\Omega_{z} \subset \overline{\Omega_{z}} \subset \Omega \backslash c_{z}$ and $\bar{D} \not \subset \Omega \backslash c_{z}$, then

$$
I_{s s m}\left(z, c_{z}, \Omega_{z}\right)=\infty .
$$

This claim implies the following theorem.

Theorem 3.5 The indicator function I(.) satisfies the two properties:

1. If $z \in \Omega \backslash \bar{D}$, then

$$
I(z)=\left|\Phi^{s}(z, z)\right| .
$$

2. If $z \in \bar{D}$, then

$$
I(z)=\infty .
$$

As a conclusion, the obstacle $D$ is characterized by the indictor function $I(\cdot)$ as follows:

$$
\bar{D}=\{z \in \Omega / I(z)=\infty\} .
$$

Proof of Theorem 3.5 We assume Claim 3.4 to be true.

1. Let $z \in \Omega \backslash \bar{D}$ and any piecewise curve $c_{z}$ in $\mathcal{C}_{z}$. For any domain $\Omega_{z}$ such that $\Omega_{z} \subset \overline{\Omega_{z}} \subset \Omega \backslash c_{z}$ the claim implies that either we have $I_{s s m}\left(z, c_{z}, \Omega_{z}\right)=\Phi^{S}(z, z)$ or $I_{s S m}\left(z . c_{z}, \Omega_{z}\right)=\infty$. Since $c_{z}$ and $\Omega_{z}$ are arbitrary then $I(z)=\left|\Phi^{s}(z, z)\right|$.

2. If $z \in \bar{D}$, then for any $c_{z}$ in $\mathcal{C}_{z}$ and any $\Omega_{z}$ such that $\Omega_{z} \subset \bar{\Omega} \backslash c_{z}$, we have $\bar{D} \not \subset \Omega \backslash c_{z}$. Hence the point (2) of the claim implies that $I_{s s m}\left(z, c_{z}, \Omega_{z}\right)=\infty$ and as a consequence $I(z)=\infty$.

\subsection{Probe method}

Consider a non-vibrating domain $\Omega$ containing the obstacle $D$ such that $\kappa^{2}$ is not a Dirichlet eigenvalue of $(-\Delta)$ on $\Omega \backslash \bar{D}$. As we mentioned in the introduction, in [8] the author proceeds in two steps to detect the obstacle from the far field. The first one is to go from the far field to the near field on $\partial \Omega$ i.e. the boundary of $\Omega$. In the second one, from this computed near field the author detects the obstacle. 
The near field is given by the Dirichlet-Neumann map:

$$
\Lambda_{D}: H^{\frac{1}{2}}(\partial \Omega) \Rightarrow H^{-\frac{1}{2}}(\partial \Omega)
$$

where $\Lambda_{D} f:=\left.\frac{\partial u^{f}}{\partial v}\right|_{\partial \Omega}, v$ on $\partial \Omega$ is oriented to outside of $\Omega$, and $u^{f} \in H^{1}(\Omega \backslash \bar{D})$ satisfies

$$
\begin{cases}\Delta u^{f}+k^{2} u^{f}=0 & \text { in } \Omega \backslash \bar{D} \\ u^{f}=f & \text { on } \partial \Omega \\ u^{f}=0 & \text { on } \partial D\end{cases}
$$

From this data, the indicator function of the probe method is related to the following quadratic form:

$$
\int_{\partial \Omega}\left(\Lambda_{D}-\Lambda_{\emptyset}\right) f(x) \cdot f(x) \mathrm{d} s(x)
$$

where $\Lambda_{\emptyset}$ is the Dirichlet-Neumann map for (3.31) when $D=\emptyset$.

For $z \in \Omega \backslash \bar{D}$, we take $c_{z}$ and $\Omega_{z}$ as in the subsection concerning the singular sources method. We approximate $\Phi(\cdot, z)$ on $\partial \Omega_{z}$ by a sequence of Herglotz waves $v_{g_{z}}$ (which is a reconstructive version of the Runge approximation used in the original probe method [7]).

Evaluating the probe functional (3.32) for $f=\left.v_{g_{n}^{z}}\right|_{\partial \Omega}$, using the Alessandrini identity on $\Omega \backslash \bar{D}$ (see, [1] or [10], Chap. 3), we find:

$$
\begin{aligned}
I_{p b}\left(z, c_{z}, \Omega_{z}\right) & :=\lim _{n \rightarrow \infty} \int_{\partial \Omega}\left(\Lambda_{D}-\Lambda_{\emptyset}\right) v_{g_{n}^{z}}(x) \cdot v_{g_{n}^{z}}(x) \mathrm{d} s(x) \\
& =-\lim _{n \rightarrow \infty} \int_{\partial D}\left(\frac{\partial v_{g_{n}^{z}}^{s}}{\partial v}(x)-\frac{\partial v_{g_{n}^{z}}^{z}}{\partial v}(x)\right) \cdot v_{g_{n}^{z}}(x) \mathrm{d} s(x),
\end{aligned}
$$

and taking the limit with respect to $n$, we find:

$$
I_{p b}\left(z, c_{z}, \Omega_{z}\right)=-\int_{\partial D}\left\{\frac{\partial \tilde{\Phi}_{\Omega}}{\partial v}(x, z)-\frac{\partial \Phi}{\partial v}(x, z)\right\} \cdot \Phi(x, z) \mathrm{d} s(x)
$$

where we denoted by $v_{g_{z}^{n}}^{S}$ and $\tilde{\Phi}$ the solutions of (3.31) replacing $f$ by $\left.v_{g_{z}}\right|_{\partial \Omega}$ and $\left.\Phi(\cdot, z)\right|_{\partial \Omega}$, respectively. The function

$$
\Phi_{\Omega}^{s}(x, z):=\tilde{\Phi}(x, z)-\Phi(x, z)
$$

is called the reflected solution for the problem (3.31). It is a solution to the problem:

$$
\begin{cases}\Delta \Phi_{\Omega}^{s}+k^{2} \Phi_{\Omega}^{s}=0 & \text { in } \Omega \backslash \bar{D} \\ \Phi_{\Omega}^{s}(\cdot, z)=0 & \text { on } \partial \Omega \\ \Phi_{\Omega}^{s}(\cdot, z)=-\Phi(\cdot, z) & \text { on } \partial D\end{cases}
$$

Using the Green's formula on $\Omega \backslash \bar{D}$ for $\Phi_{\Omega}^{s}(\cdot, z)$ and $\Phi(\cdot, z)$ we deduce that

$$
I_{p b}\left(z, c_{z}, \Omega_{z}\right)=-\Phi_{\Omega}^{s}(z, z)+\int_{\partial \Omega} \frac{\partial}{\partial v} \Phi_{\Omega}^{s}(x, z) \Phi(x, z) \mathrm{d} s(x) .
$$


We are interested with $z$ in $\Omega \backslash \bar{D}$. Since $\Phi_{\Omega}^{s}(\cdot, z)+\Phi(\cdot, z)$ satisfies

$$
\begin{cases}\left(\Delta+k^{2}\right)\left(\Phi_{\Omega}^{s}(\cdot, z)+\Phi(\cdot, z)\right)=-\delta(\cdot-z) & \text { in } \Omega \backslash \bar{D} \\ \Phi_{\Omega}^{s}(\cdot, z)+\Phi(\cdot, z)=\Phi(\cdot, z), & \text { on } \partial \Omega, \\ \Phi_{\Omega}^{s}(\cdot, z)+\Phi(\cdot, z)=0, & \text { on } \partial D\end{cases}
$$

then the function $\Phi_{\Omega}^{s}(\cdot, z)+\Phi(\cdot, z)$ can be seen as a sum of the Dirichlet Green's function of $\Delta+k^{2}$ on $\Omega \backslash \bar{D}$ and the solution of (3.34) replacing $\delta$ by zero. Since both of these two functions are bounded with respect to $z$, in $\Omega \backslash \bar{D}$ and near $\partial D$, with values in $C^{1}(\partial \Omega)$, we deduce that

$$
\int_{\partial \Omega} \frac{\partial}{\partial v} \Phi_{\Omega}^{S}(x, z) \Phi(x, z) \mathrm{d} s(x)
$$

is bounded with respect to $z$. This means that

$$
\left|I_{p b}\left(z, c_{z}, \Omega_{z}\right)+\Phi_{\Omega}^{s}(z, z)\right|=O(1) \quad \text { for } z \quad \text { in } \Omega \backslash \bar{D} .
$$

Let us now compare $\Phi_{\Omega}^{s}(z, z)$ with $\Phi^{s}(z, z)$. We set $\Psi(x, z):=\Phi_{\Omega}^{s}(x, z)+\Phi^{s}(x, z)$. Then $\Psi(\cdot, z)$ satisfies:

$$
\begin{cases}\Delta \Psi+k^{2} \Psi=0 & \text { in } \Omega \backslash \bar{D} \\ \Psi(\cdot, z)=\Phi^{s}(\cdot, z) & \text { on } \partial \Omega, \\ \Psi(\cdot, z)=0 & \text { on } \partial D .\end{cases}
$$

For $z$ in $\Omega \backslash \bar{D}$ and near $\partial D, \Phi^{s}(\cdot, z)$ is bounded in $C^{2}(\partial \Omega)$. We can see it by remarking that $\Phi^{s}(x, z)+\Phi(x, z)$ is the Dirichlet Green's function of our equation on $\mathbb{R}^{3} \backslash \bar{D}$. Then the wellposedness of the problem (3.31) implies that $\Psi(\cdot, z)$ is bounded in $C^{2}(\Omega)$ hence $\Psi(z, z)$ is bounded. This means that

$$
\left|\Phi_{\Omega}^{S}(z, z)+\Phi^{S}(z, z)\right|=O(1) \text { for } z \text { in } \Omega \backslash \bar{D} \text { and near } \partial D .
$$

As we mentioned in the paragraph after (3.32) the indicator function of the original probe method, i.e. $I_{p b}\left(z, c_{z}, \Omega_{z}\right)$, is constructed from the Dirichlet-Neumann map by using a sequence of functions approximating the fundamental solution and the Alessandrini identity.

Now, in place of using the Dirichlet to Neumann map as a starting point, we use the far field data $u^{\infty}(\theta, d),(\theta, d) \in \mathbb{S}^{2}$. Using the same sequence of functions approximating the fundamental solution and the identity (2.12), which is the far field counter part of the Alessandrini identity, we end up with

$$
I_{s s m}\left(z, c_{z}, \Omega_{z}\right)=\Phi^{s}(z, z) .
$$

In addition, we have the following proposition

\section{Proposition 3.6}

$$
\left|I_{p b}\left(z, c_{z}, \Omega_{z}\right)-I_{s s m}\left(z, c_{z}, \Omega_{z}\right)\right|=O(1),
$$

for $z$ in $\Omega \backslash \bar{D}$ and near $\partial D$.

Proof of Proposition 3.6 From Proposition 3.1 and the combination of (3.35) and (3.37) we get

$$
\left|I_{p b}\left(z, c_{z}, \Omega_{z}\right)-I_{s s m}\left(z, c_{z}, \Omega_{z}\right)\right|=\left|I_{p b}\left(z, c_{z}, \Omega_{z}\right)-\Phi^{s}(z, z)\right|=O(1) .
$$


This proposition and the comments before suggest that the natural far field version of the probe method is the one given in Definition 3.2.

\subsection{Conclusion and some comments}

From the singular sources method in Sect. 3.1 and the probe method in Sect. 3.2, we deduce the following theorem.

Theorem 3.7 The natural far field versions of the probe method and the singular sources method are identical. This common version is given by Definition 3.2.

1. The full convergence proof of this natural far field version is not achieved yet. The first point of the claim is shown to be true, see (2.25) and we have $\Phi^{S}(z, z) \rightarrow \infty$ when $z \rightarrow \partial D$ (see [18]). The justification of the second point of the claim is not yet proven. A partial result in this direction is the recent work [9], where the obstacle boundary value problem is considered. It is proved under some assumptions on the smallness of the frequency $\kappa$.

2. For the method based on the indicator given Definition 3.2, we need to take some domain $\Omega$ containing the unknown obstacle. This is also the case for the linear sampling and the factorization methods. But such domain $\Omega$ can always be found using the first version of the no response test. Indeed, it is given by testing if for any $\Omega$ we have $I_{1}(\Omega)=0$. This shows how the combination of these methods can be useful.

3. The way of defining the set $\Omega_{z}$ in Definition 3.2 is nothing but the needle approach introduced in [7].

\section{The second version of the no-response test}

In this section, we develop the second version of the no-response test which combines the superposition principle and the range test idea introduced in [19].

Again, consider a bounded domain $B \subset \mathbb{R}^{3}$ with boundary of class $C^{2}$. The basic idea of the range test is to test the solvability of the equation

$$
\frac{1}{4 \pi} \int_{\partial B} \mathrm{e}^{-i \kappa \theta \cdot x} \psi(x) \mathrm{d} s(x)=u^{\infty}(\theta, d), \quad(\theta, d) \in \mathbb{S} \times \mathbb{S} .
$$

Here, we will use this technique applied to the far field pattern $v_{g}^{\infty}$ of the Herglotz wave functions used in the definition of the no response test above.

For regularization of the ill-posed integral Eq. (4.38) we use the Tikhonov regularization scheme

$$
\psi_{\alpha}:=\left(\alpha+S^{\infty, *} S^{\infty}\right)^{-1} S^{\infty, *} u^{\infty}
$$

with regularization parameter $\alpha>0$ and the far field operator $S^{\infty}: L^{2}(\partial B) \rightarrow L^{2}(\mathbb{S})$ is given by

$$
\left(S^{\infty} \varphi\right)(\theta):=\frac{1}{4 \pi} \int_{\partial B} \mathrm{e}^{-i \kappa \theta \cdot y} \varphi(y) \mathrm{d} s(y), \quad \theta \in \mathbb{S} .
$$


Definition 4.1 (The second version of the no-response method) For a non-vibrating domain $B$ we define the indicator function

$$
\begin{aligned}
& I_{2}(B):=\lim _{\epsilon \rightarrow 0} \sup \left\{\lim _{\alpha \rightarrow 0}\left\|\psi_{g}^{\alpha}\right\|_{L^{2}(\partial B)}: \quad \psi_{g}^{\alpha}\right. \text { is the regularized solution (4.39) } \\
&\text { of (4.38) with } \left.u^{\infty}=v_{g}^{\infty}, g \in L^{2}(\mathbb{S}) \text { and }\left\|v_{g}\right\|_{L^{2}(\partial B)}<\epsilon\right\}
\end{aligned}
$$

For the set $\mathcal{G}$ of non-vibrating domains $B$, this second version of no response test calculates the indicator function $I_{2}(B)$ and builds the intersection

$$
D_{\text {rec }, 2}:=\bigcap_{B \in \boldsymbol{B}_{2}} B
$$

where

$$
\boldsymbol{B}_{2}:=\left\{B \in \mathcal{G}: I_{2}(B)=0\right\} .
$$

The convergence of the no response test is given by the following result.

Theorem 4.2 (Convergence of the second version) Let $\mathcal{G}$ be as in Definition 4.1. We have

1. If $\bar{D} \subset B$ then $I_{2}(B)=0$.

2. If $\bar{D} \not \subset B$ then $I_{2}(B)=\infty$.

Thus, the obstacle D can be characterized by

$$
\bar{D}=D_{\text {rec }, 2} \text {. }
$$

The following lemmas are the key tools to prove Theorem 4.2. The proof of the first one can be found in [6] and [19].

Lemma 4.3 Let $B$ be a domain with boundary of class $C^{2}$. We consider an injective integral operator with continuous kernel and dense range

$$
(A \psi)(\theta):=\int_{\partial B} k(\theta, y) \psi(y) d s(y), \quad \theta \in \mathbb{S}
$$

from $L^{2}(\partial B)$ to $L^{2}(\mathbb{S})$. Then the Tikhonov regularized solution of the equation $A \psi=f$ given by

$$
\psi_{\alpha}:=\left(\alpha I+A^{*} A\right)^{-1} A^{*} f
$$

where $\alpha$ is the regularized parameter and $A^{*}$ is the adjoint of $A$ satisfies

$$
\lim _{\alpha \rightarrow 0}\left\|\psi_{\alpha}\right\|_{L^{2}(\partial B)}= \begin{cases}\infty, & \text { if } f \text { is not in } A\left(L^{2}(\partial B)\right), \\ \left\|\psi^{*}\right\|_{L^{2}(\partial B)}, & \text { if } A \psi^{*}=f .\end{cases}
$$

From Rellich's Lemma we immediately obtain the following result.

Lemma 4.4 If the Eq. (4.38) is solvable, i.e. there exists $\psi \in L^{2}(\partial B)$ such that

$$
S^{\infty} \psi=u^{\infty}
$$


then the scattered field $u^{s}$ of $u^{\infty}$ is given by

$$
u^{s}=\int_{\partial B} \Phi(\cdot, y) \psi(y) \mathrm{d} s(y) \text { in } \mathbb{R}^{3} \backslash(B \cup D) .
$$

Also, we collect basic mapping properties of the single-layer operator.

Lemma 4.5 The operator $S: L^{2}(\partial B) \rightarrow H^{1}(\partial B)$ defined by:

$$
(S \psi)(x):=\int_{\partial B} \Phi(x, y) \psi(y) \mathrm{d} s(y), \quad x \in \partial B
$$

is an isomorphism if $B$ is a non-vibrating domain.

Proof of Lemma 4.5 In [12], Theorem 7.17, it is proved that for such regular domains $B$ the operator $S: L^{2}(\partial B) \rightarrow H^{1}(\partial B)$ is Fredholm with index zero. The injectivity and hence the surjectivity of $S$ is given by the assumption that $B$ is a non-vibrating domain.

Lemma 4.6 The operator $S^{\infty}: L^{2}(\partial B) \rightarrow L^{2}(\mathbb{S})$ has a dense range if $B$ is a nonvibrating domain.

Proof of Lemma 4.6 We define $\mathcal{F}_{B}: H^{\frac{1}{2}}(\partial B) \rightarrow L^{2}(\mathbb{S})$ to be the far field map for the artificial obstacle $B$, i.e for $u \in H^{\frac{1}{2}}(\partial B), \mathcal{F}_{B} u$ is the far field of the solution $u^{s}$ of the $\left(\Delta+\kappa^{2}\right) u^{s}=0$ satisfying the Sommerfeld radiation condition and $u^{s}=u$ on $\partial B$.

In terms of the isomorphism $S: H^{-\frac{1}{2}}(\partial B) \rightarrow H^{\frac{1}{2}}(\partial B)$, see [12], we can write $S^{\infty}=\mathcal{F}_{B} S$. We set also the Herglotz wave operator $H: L^{2}(\mathbb{S}) \rightarrow H^{\frac{1}{2}}(\partial B), H g:=\left.v_{g}\right|_{\partial B}$ where $v_{g}$ is the Herglotz wave function (2.5). We denote by $H^{*}$ its dual operator from $H^{-\frac{1}{2}}(\partial B) \rightarrow L^{2}(\mathbb{S})$. Let $\phi \in L^{2}(\partial B)$. By (4.40), we have

$$
H^{*} \phi=\int_{\partial B} \phi(x) e^{-i k d \cdot x} \mathrm{~d} s(x)=4 \pi S^{\infty} \phi
$$

which means that

$$
S^{\infty} \phi=\mathcal{F}_{B} S \phi=\frac{1}{4 \pi} H^{*} \phi
$$

for every $\phi \in L^{2}(\partial B)$ and hence for every $\phi \in H^{-\frac{1}{2}}(\partial B)$ by the continuity of $\mathcal{F}_{B}, S$, $H^{*}$ and the denseness of $L^{2}(\partial B)$ in $H^{-\frac{1}{2}}(\partial B)$.

Now since $B$ is a non-vibrating domain then $H$ is injective and hence $H^{*}$ has a dense range. From (4.48), we see that the operator $S^{\infty}$ stated on $H^{-\frac{1}{2}}(\partial B)$ has a dense range. Finally, $S^{\infty}$ stated on $L^{2}(\partial B)$ has a dense range, because $L^{2}(\partial B)$ is dense in $H^{-\frac{1}{2}}(\partial B)$ and $S^{\infty}$ is continuous on $H^{-\frac{1}{2}}(\partial B)$ (since $\mathcal{F}_{B}: H^{\frac{1}{2}}(\partial B) \rightarrow L^{2}(\mathbb{S})$ and $S: H^{-\frac{1}{2}}(\partial B) \rightarrow H^{\frac{1}{2}}(\partial B)$ are continuous).

Proof of Theorem 4.2 We will investigate the two cases $\bar{D} \subset B$ and $\bar{D} \not \subset B$ in two steps.

$I$ Case 1 Consider the case when $\bar{D} \subset B$. We take any $g \in L^{2}(\mathbb{S})$ satisfying $\left\|v_{g}\right\|_{L^{2}(\partial B)}<\epsilon$. As for the case one of the first version, using the regularity of very week solution for elliptic problems and interior estimates, this implies that

$$
\left\|v_{g}\right\|_{C^{1}(\partial D)}<c \epsilon
$$


with some positive constant $c$. Since $\bar{D} \subset B$ the scattered field $v_{g}^{s}$ has a trace on $\partial B$ which is in $C^{1}(\partial B)$ and $\left\|v_{g}^{s}\right\|_{C^{1}(\partial B)}<C \epsilon$ with some appropriate constant $C$ depending on the scatterer and on $\partial B$. In this case, by lemma 4.5 the single-layer equation

$$
S \psi=v_{g}^{s} \quad \text { on } \partial B
$$

has a solution $\psi \in L^{2}(\partial B)$ which satisfies

$$
\|\psi\|_{L^{2}(\partial B)} \leq \tilde{c} \epsilon
$$

with a further constant $\tilde{c}$. Hence, also the Eq. (4.38) with $u^{\infty}=v_{g}^{\infty}$ is solvable with $\psi$ as the solution and we obtain $I_{2}(B)=0$.

II Case 2 Assume that $\bar{D} \not \subset B$. II.A) We first assume that $D \not \subset \bar{B}$. Then, there is a point $z \in \partial D \backslash \bar{B}$. We choose some arbitrary $\epsilon>0$. As in section 2, we take a sequence of points $z_{p} \in \mathbb{R}^{3} \backslash(\overline{D \cup B})$ such that $z_{p} \rightarrow z$ and construct Herglotz wave functions which approximate $\epsilon / 2 \cdot \alpha_{p}$ times the point source $\Phi\left(\cdot, z_{p}\right)$ in $L^{2}\left(\partial B_{p}\right)$ where $\overline{D \cup B} \subset B_{p}$ and $z_{p} \notin B_{p}$. We recall that $\alpha_{p}:=\left(\left\|\Phi\left(\cdot, z_{p}\right)\right\|_{L^{2}(\partial B)}\right)^{-1}$. Also, using the well-posedness of the scattering problem we obtain that

$$
v_{g_{n}^{p}}^{\infty} \rightarrow \frac{\epsilon}{2} \alpha_{p} \Phi^{\infty}\left(\cdot, z_{p}\right), \quad n \rightarrow \infty
$$

in $L^{2}(\mathbb{S})$.

Next, we need to consider the solvability of the Eq. (4.38) with right-hand side $u^{\infty}=v_{g_{n}^{p}}^{\infty}$. Here, we will distinguish two possibilities:

II.A. $\alpha$ There exists a couple $\left(p_{0}, n_{0}\right)$ such that $\left\|v_{g_{n} p_{0}}\right\|_{L^{2}(\partial B)}<\epsilon$ and (4.38) is not solvable. In this case by the Tikhonov regularization, we find a regularized sequence of solutions $\psi_{g n_{0}}^{\alpha}$ of (4.38) such that $\left\|\psi_{g_{n_{0}}^{p_{0}}}^{\alpha}\right\|_{L^{2}(\partial B)}$ tends to $\infty$ as $\alpha$ tends to zero, where $\alpha$ is the regularization parameter. Hence we obtain the desired statement.

II.A. $\beta$ For every couple $(p, n)$ such that $\left\|v_{g_{n}^{p}}\right\|_{L^{2}(\partial B)}<\epsilon$ the Eq. (4.38) is solvable. By Lemma 4.4 for every such couple $(p, n), v_{g_{n}^{p}}^{n}$ is extendable up to $\partial B$ such that its trace is in $H^{1}(\partial B)$. Now solving the equation (4.50) with right-hand side $v_{g_{n}^{p}}^{s}$ we get a sequence of solutions $\psi_{g_{n}^{p}}$ of (4.38), with right-hand side given by $v_{g_{n}^{p}}^{\infty}$ i.e.

$$
S^{\infty} \psi_{n}^{p}=v_{g_{n}^{p}}^{\infty} .
$$

We distinguish two cases. The first one is that there exists $p_{0}$ such that the sequence $\left\|\psi_{g_{n}{ }_{n}}\right\|_{L^{2}(\partial B)}$ is unbounded. In this case we obtain the desired statement. The second case is that for every $p$ the sequence $\left\|\psi_{g_{n}}\right\|_{L^{2}(\partial B)}$ is bounded. In this case for every $p$ fixed we can find a function $\psi_{p} \in L^{2}(\partial B)$ such that $\psi_{g_{n}}$ tends weakly to $\psi_{p}$ in $L^{2}(\partial B)$. For every $p$ fixed, we take the limit in (4.53) with respect to $n$. We obtain,

$$
S^{\infty} \psi_{p}=\frac{\epsilon}{2} \alpha_{p} \Phi^{\infty}\left(\cdot, z_{p}\right)
$$

on $\mathbb{S}$. This means that (4.38) is solvable for $u^{\infty}$ being the far field pattern of $\Phi^{S}\left(\cdot, z_{p}\right)$. Hence using again Lemma 4.4, we deduce that $\Phi^{S}\left(\cdot, z_{p}\right)$ is extendable up to $\partial B$ with boundary values in $H^{1}(\partial B)$. The solution of (4.54) is then given by the solution of

$$
S \psi_{p}=\frac{\epsilon}{2} \alpha_{p} \Phi^{s}\left(\cdot, z_{p}\right) \quad \text { on } \partial B .
$$


In this case the sequence $\alpha_{p}$ is bounded from below by a positive constant, because $\left(z_{p}\right)_{p \in \mathbb{N}}$ is in $\partial D \backslash \bar{B}$. We show that $\Phi^{s}\left(\cdot, z_{p}\right)$ is unbounded in $H^{1}(\partial B)$. Indeed suppose that $\left\|\Phi^{S}\left(\cdot, z_{p}\right)\right\|_{H^{1}(\partial B)}$ is bounded. Since $\Phi^{S}\left(x, z_{p}\right)$ satisfies $-\Delta \Phi^{S}\left(x, z_{p}\right)+\kappa^{2} \Phi^{S}\left(x, z_{p}\right)=$ 0 in $\mathbb{R}^{3} \backslash \bar{B}$ then $\Phi^{s}\left(\cdot, z_{p}\right)$ is bounded in $H_{l o c}^{\frac{3}{2}}\left(\mathbb{R}^{3} \backslash \bar{B}\right)$ hence also in $H^{1}\left(\partial D \cap\left(\mathbb{R}^{3} \backslash \bar{B}\right)\right)$. But $\Phi^{S}\left(\cdot, z_{p}\right)=\Phi\left(\cdot, z_{p}\right)$ on $\partial D$ and $\Phi\left(\cdot, z_{p}\right)$ in unbounded in $H^{1}\left(\partial D \cap\left(\mathbb{R}^{3} \backslash \bar{B}\right)\right)$. This gives a contradiction.

II.B) We now assume that $D \subset B$. From $\bar{D} \not \subset B$ we obtain that there is a point $z \in \partial B \cap \partial D$. Let $z_{p}$ be a sequence of points in $\mathbb{R}^{3} \backslash \overline{(B \cup D)}$ tending to $z$. Applying the Green's Theorem on $\mathbb{R}^{3} \backslash \bar{B}$ for $\Phi^{S}\left(\cdot, z_{p}\right)$ and $\Phi\left(\cdot, z_{p}\right)$, we get

$$
\alpha_{p} \int_{\partial B} \Phi^{s}\left(\cdot, z_{p}\right) \frac{\partial \Phi}{\partial v}\left(\cdot, z_{p}\right) d s(x)-\alpha_{p} \int_{\partial B} \Phi\left(\cdot, z_{p}\right) \frac{\partial \Phi^{s}}{\partial v}\left(\cdot, z_{p}\right) \mathrm{d} s(x)=\alpha_{p} \Phi^{s}\left(z_{p}, z_{p}\right) .
$$

Hence if $\alpha_{p} \Phi^{s}\left(\cdot, z_{p}\right)$ is bounded in $H^{1}(\partial B)$ then $\alpha_{p} \frac{\partial \Phi^{s}}{\partial v}\left(\cdot, z_{p}\right)$ is also bounded in $L^{2}(\partial B)$. Using the estimate

$$
\left\|\frac{\partial \Phi}{\partial v}\left(\cdot, z_{p}\right)\right\|_{H^{-1}(\partial B)} \leq\left\|\Phi\left(\cdot, z_{p}\right)\right\|_{L^{2}(\partial B)}
$$

the second member of (4.56) behaves like $\left\|\Phi\left(\cdot, z_{p}\right)\right\|_{L^{2}(\partial B)} \approx\left(\ln \left(d\left(z_{p}, \partial B\right)\right)\right)^{\frac{1}{2}}$. This fact and the estimate

$$
\Phi^{S}\left(z_{p}, z_{p}\right)=O\left[\frac{1}{d\left(z_{p}, \partial B\right)}\right]
$$

(see Theorem 2.1.15 of [18]) imply that the left hand side of (4.56) is of the order $O\left(\sqrt{\ln \left(d\left(z_{p}, \partial B\right)\right)}\right)$ while the right hand side behaves as $O\left(\frac{1}{d\left(z_{p}, \partial B\right)}\right)$, which is impossible. Thus, we have

$$
\left\|\alpha_{p} \Phi^{S}\left(\cdot, z_{p}\right)\right\|_{H^{1}(\partial B)} \rightarrow \infty, \quad p \rightarrow \infty .
$$

Then, also $\psi_{p}=\frac{\epsilon}{2} \alpha_{p} S^{-1} \Phi^{S}\left(\cdot, z_{p}\right)$ cannot be bounded in $L^{2}(\partial B)$.

Finally in all these cases, we constructed sequences $g_{n}^{p}$ such that $\left\|v_{g_{n}^{p}}\right\|_{L^{2}(\partial B)}<\epsilon$ and $\lim _{\alpha \rightarrow 0}\left\|\psi_{g_{n}^{p}}^{\alpha}\right\|_{L^{2}(\partial D)}$ is unbounded. This means that $I_{2}(B)=\infty$.

As for the proof of Theorem 2.2 the properties 1 . and 2. imply that $\bar{D}=D_{\text {rec }, 2}$.

Remark 4.7 In the definition of the two versions of the no-response test, we can replace in (2.13) and (4.41) the $L^{2}(\partial B)$ norm by the $C^{1}(\partial B)$ norm. For these changes, the convergence of these two methods is as follows:

1. If $D \subset B$ then $I_{1}(B)=I_{2}(B)=0$.

2. If $D \not \subset B$ then $I_{1}(B)=I_{2}(B)=\infty$.

The difference with the original versions is that $\bar{D} \subset B$ implies that $I_{1}(B)=I_{2}(B)=$ 0 . This means that if $\partial D \not \subset B$ then $I_{1}(B)=I_{2}(B)=0$ which is not the case for original versions we gave. This is due to (2.12) and (4.50) respectively and the fact that the $C^{1}(\partial B)$-norm estimate of $v_{g}$ implies the $C^{1}(\partial B)$-norm estimate of $v_{g}^{s}$. 


\section{Convergence of the linear sampling method implies the convergence of the no response method}

In this section we recall the linear sampling method and show how its convergence implies the convergence of the second version of the no response test.

The linear sampling method The fundamental object of the linear sampling method is the following linear integral operator $F: L^{2}(\mathbb{S}) \rightarrow L^{2}(\mathbb{S})$, given by

$$
F g(\theta):=\int_{\mathbb{S}} u^{\infty}(\theta, d) g(d) \mathrm{d} s(d), \quad \theta \in \mathbb{S} .
$$

This operator is called the far-field operator. Let $g \in L^{2}(\mathbb{S})$ and $v_{g}:=\int_{\mathbb{S}} \mathrm{e}^{i \kappa d \cdot x} g(\theta) \mathrm{d} s(\theta)$, $x \in \mathbb{R}^{3}$. From the asymptotic behavior of this fundamental solution we know that the far-field of $\Phi(\cdot, z), z \in \mathbb{R}^{3}$, is given by

$$
\Phi_{\infty}(\theta, z)=\frac{1}{4 \pi} \mathrm{e}^{-i \kappa \theta \cdot z}, \quad \theta \in \mathbb{S} .
$$

When $z \in D$, then $\Phi_{\infty}(\hat{x}, z)=\Phi^{\infty}(\hat{x}, z)$, where we used $\Phi^{\infty}(\hat{x}, z)$ to be the far field pattern of the scattered field $\Phi^{S}(x, z)$ created by the obstacle $D$ using $\Phi(x, z)$ as the incident wave.

The idea of the linear sampling method is to approximately solve the following integral equation, called the far field equation:

$$
F g_{z}=\Phi_{\infty}(\cdot, z)
$$

for a grid of points $z$ and to look at the behavior of the norms of $g_{z}$. It is observed that these norms blow up near and outside $\partial D$. The behavior of the norms of $g_{z}$ given for a grid of points $z$ is used to localize $D$.

A detailed version is given in the following theorem, see [4].

Theorem 5.1 Assume that $\kappa^{2}$ is not a Dirichlet eigenvalue of $-\Delta$ in D. We have

1. If $z \in D$, then for every $\epsilon>0$ there exists a solution $g^{\epsilon}(\cdot, z)$ in $L^{2}(\mathbb{S})$ of the inequality

$$
\left\|F g^{\epsilon}(\cdot, z)-\Phi^{\infty}(\cdot, z)\right\|_{L^{2}(\mathbb{S})}<\epsilon
$$

such that

$$
\lim _{z \rightarrow \partial D}\left\|g^{\epsilon}(\cdot, z)\right\|_{L^{2}(\mathbb{S})}=\lim _{z \rightarrow \partial D}\left\|v_{g^{\epsilon}}(\cdot, z)\right\|_{H^{1}(D)}=\infty
$$

2. If $z \in \mathbb{R}^{3} \backslash \bar{D}$, then for every $\epsilon>0$ and $\delta>0$ there exists a solution $g^{\epsilon, \delta}(\cdot, z)$ in $L^{2}(\mathbb{S})$ of the inequality

$$
\left\|F g^{\epsilon, \delta}(\cdot, z)-\Phi^{\infty}(\cdot, z)\right\|_{L^{2}(\mathbb{S})}<\epsilon+\delta
$$

such that

$$
\lim _{\delta \rightarrow 0}\left\|g^{\epsilon, \delta}(\cdot, z)\right\|_{L^{2}(\mathbb{S})}=\lim _{\delta \rightarrow 0}\left\|v_{g^{\epsilon, \delta}}(\cdot, z)\right\|_{H^{1}(D)}=\infty .
$$


Using the density $g$ of the linear sampling method for the second version To prove the convergence of the linear sampling method one has to assume that $\kappa^{2}$ is not a Dirichlet eigenvalue for $-\Delta$ on $D$, i.e $D$ is a non-vibrating domain. With this assumption, from Theorem 5.1 we have a sequence $g^{\epsilon}$ which creates the blow-up. Using this sequence $g^{\epsilon}$ we will now justify the blow-up in the case two of the second version of the no-response test. As we will see, the arguments we use do not depend on the type of equation nor the type of obstacle (penetrable or not). This means that in any case where the linear sampling method converges then the no-response converges too.

Let us now explain how we can use the singular sequence $g^{\epsilon}$ of the linear sampling method to create the blow-up for the no-response test. To this end we will go into the explicit construction of the sequence as carried out in [4] and use its properties. Note that for some general solution $g^{\epsilon}$ of (5.59) it is not yet proven that it will coincide with this particular solution whose existence is stated by the above theorem. However, our assumption will be that the linear sampling method is convergent in the sense that it picks this particular solution. For more precision about this fact and the mathematical justification of the linear sampling method, we refer the reader to the work [3].

Theorem 5.2 Consider a non-vibrating domain $B$ such that $\bar{D} \not \subset B$. Given the densities $g^{\epsilon}(\cdot, z)$ provided by Theorem 5.1 as the basic ingredient for the indicator function of the linear sampling method, there is a density $\tilde{g}(\cdot, z)$ such that the Herglot $z$ wave function $v_{\tilde{g}(\cdot, z)}$ is bounded in a neighbourhood of $z$ and the density $g_{R T}(\cdot, z):=g^{\epsilon}(\cdot, z)+\tilde{g}(\cdot, z)$ leads to a blow-up of the functional $I_{2}$ of the second version of the no-response test.

Remark The modification by $\tilde{g}$ is necessary only to tailor the Herglotz wave function of the linear sampling method to the normalization assumptions on $B$ demanded by the no-response test. Alternatively, we could just neglect the normalization assumption of the no response test and feed the density $g^{\epsilon}(\cdot, z)$ into the functional $I_{1, \epsilon}$ defined in (2.14). We consider this to be an interesting question for the further analysis of the linear sampling method in its connection to the no response test.

Proof By assumption we have a non-vibrating domain $B$ such that $\bar{D} \not \subset B$. The situation $\bar{D} \not \subset B$ means that either $\partial D \backslash \bar{B} \neq \emptyset$ or $\partial D \subset \bar{B}$.

I. We consider first the case where $\partial D \backslash \bar{B} \neq \varnothing$. For this case, we may choose a point $a \in \partial D \backslash \bar{B}$ and a sequence $z_{p} \rightarrow a$ for $p \rightarrow \infty$ with $z_{p} \in D$. From the part 1$)$ of Theorem 5.1, there is a sequence $g^{\epsilon}\left(\cdot, z_{p}\right)$ such that we have

$$
\left\|F g^{\epsilon}\left(\cdot, z_{p}\right)-\Phi^{\infty}\left(\cdot, z_{p}\right)\right\|_{L^{2}(\mathbb{S})}<\epsilon,
$$

and

$$
\lim _{z_{p} \rightarrow a}\left\|v_{g^{\epsilon}}\left(\cdot, z_{p}\right)\right\|_{H^{1}(D)}=\infty .
$$

In [4], page 416, the sequence $v_{g^{\epsilon}\left(\cdot, z_{p}\right)}$ is constructed such that it tends to some single layer potential

$$
S \phi_{z_{p}}(x):=\int_{\partial D} \Phi(x, y) \phi_{z_{p}}(y) \mathrm{d} s(y), \quad x \in \partial D,
$$

in $H^{\frac{1}{2}}(\partial D)$ where $\phi_{z_{p}}$ is the solution of the integral equation $S \phi_{z_{p}}(x)=-\Phi\left(x, z_{p}\right)$.

Let us now consider the sequence $\left(v_{g^{\epsilon}}^{\infty}\left(\cdot, z_{p}\right)\right)_{p \in \mathbb{N}}$. Taking this sequence as the right side of (4.38) either the Eq. (4.38) is not solvable for some $p_{0}$ and hence its regularized solution $\psi_{p_{0}, \alpha}^{\epsilon}$ satisfies 


$$
\lim _{\alpha \rightarrow 0}\left\|\psi_{p_{0}, \alpha}^{\epsilon}\right\|_{L^{2}(\partial B)}=\infty
$$

or (4.38) with right-side $v_{g^{\epsilon}}^{\infty}\left(\cdot, z_{p}\right)$ is solvable for every $p$ and then via Lemma 4.4 the scattered fields $v_{g^{\epsilon}}^{s}\left(\cdot, z_{p}\right)$ will be extendable up to $\partial B$ such that its trace is in $H^{1}(\partial B)$. Now the corresponding sequence of solutions $\psi_{p}^{\epsilon}$ of (4.38) satisfies the equation

$$
\int_{\partial B} \Phi(x, y) \psi_{p}^{\epsilon}(y) \mathrm{d} s(y)=v_{g^{\epsilon}}^{s}\left(x, z_{p}\right) \quad \text { on } \partial B
$$

Next, we will prove that the sequence $v_{g^{\epsilon}}^{S}\left(\cdot, z_{p}\right)$ is not bounded in $H^{\frac{1}{2}}(\partial B)$ (and hence also in $\left.H^{1}(\partial B)\right)$. Indeed, suppose that the sequence $\left(v_{g^{\epsilon}}^{s}\left(\cdot, z_{p}\right)\right)_{p \in \mathbb{N}}$ is bounded in $H^{\frac{1}{2}}(\partial B)$. The wellposedness of the forward scattering problem on $\mathbb{R}^{3} \backslash B$ implies that $\left(v_{g^{\epsilon}}^{s}\left(\cdot, z_{p}\right)\right)_{p \in \mathbb{N}}$ is bounded in $H_{l o c}^{1}\left(\mathbb{R}^{3} \backslash B\right)$ hence $\left.v_{g^{\epsilon}}\left(\cdot, z_{p}\right)\right|_{\partial D \cap \mathcal{V}(a)}(=$ $\left.-\left.v_{g^{\epsilon}}^{s}\left(\cdot, z_{p}\right)\right|_{\partial D \cap \mathcal{V}(a)}\right)$, where $\mathcal{V}(a)$ is a neighborhood of $a$ such that $\mathcal{V}(a) \cap \bar{B}=\emptyset$, is bounded in $L^{2}(\partial D \cap \mathcal{V}(a))$. We recall that $v_{g^{\epsilon}}\left(\cdot, z_{p}\right)$ approximates $S \phi_{z_{p}}$ in $H^{\frac{1}{2}}(\partial D)$, hence also in $L^{2}(\partial D \cap \mathcal{V}(a))$. Since $S \phi_{z_{p}}=-\Phi\left(\cdot, z_{p}\right)$ on $\partial D$, we deduce that the sequence $\left(\Phi\left(\cdot, z_{p}\right)\right)_{p \in \mathbb{N}}$ is bounded in $L^{2}(\partial D \cap \mathcal{V}(a))$, which is not true. Then the sequence $\left(v_{g_{\epsilon}}^{S}\left(\cdot, z_{p}\right)\right)_{p \in \mathbb{N}}$ is unbounded in $H^{\frac{1}{2}}(\partial B)$.

From (5.62), the sequence of solutions, $\psi_{p}^{\epsilon}$, satisfies

$$
\lim _{p \rightarrow \infty}\left\|\psi_{p}^{\epsilon}\right\|_{L^{2}(\partial B)}=\infty
$$

Normalization of the sequence $v_{g \epsilon}\left(\cdot, z_{p}\right)$. To finish the proof for this case, we need to normalize the sequence $v_{g^{\epsilon}}\left(\cdot, z_{p}\right)$, i.e. to have $\left\|v_{g^{\epsilon}}\left(\cdot, z_{p}\right)\right\|_{L^{2}(\partial B)} \leq \epsilon$. It is enough to prove that this sequence is bounded. Hence, since every step in the argument is linear, multiplying it by $\epsilon$ we get the desired property.

We start by proving that $v_{g^{\epsilon}}\left(\cdot, z_{p}\right)$ is bounded in $H^{1}(D \cap K)$, for any $C^{2}$-regular domain $K$ not containing a neighborhood of the point $a$. Indeed, The function $W_{z_{p}}:=S \phi_{z_{p}}+\Phi\left(\cdot, z_{p}\right)$ satisfies

$$
\begin{cases}\Delta W_{z}+\kappa^{2} W_{z}=-\delta(z) & \text { in } D \\ W_{z}=0 & \text { on } \partial D\end{cases}
$$

i.e $W_{z}$ is the Green's function on $D$. From the estimates of this Green's function, we deduce that the sequence $\left\|W_{z_{p}}\right\|_{H^{1}(D \cap K)}, p \in \mathbb{N}$, is bounded. Hence $S \phi_{z_{p}}$ also has the same property since the sequence $\Phi\left(\cdot, z_{p}\right)$ does. This implies that for $\epsilon$ fixed the sequence $\left\|v_{g^{\epsilon}\left(\cdot, z_{p}\right)}\right\|_{H^{1}(D \cap K)}$ is bounded.

Let us now consider its $L^{2}(\partial B)$-norm. The only information we know is that $\left\|v_{g^{\epsilon}}\left(\cdot, z_{p}\right)-S \phi_{z_{p}}\right\|_{H^{1}(D)} \leq \epsilon$ and we have no information on the behavior of the sequence $\left(g^{\epsilon}\left(\cdot, z_{p}\right)\right)_{p}$ in $B \backslash \bar{D}$. Thus we cannot affirm its boundedness in $L^{2}(\partial B)$. For this reason, we modify it by another sequence which has this property and which behaves as $v_{g^{\epsilon}}\left(\cdot, z_{p}\right)$ near the point $a$.

Let $E$ be any $C^{2}$-regular domain containing $D \cup B$ such that $\mathcal{V}(a) \subset \partial E \cap(\partial D \backslash B)$. We also assume that $E$ is a non-vibrating domain. We take any $C^{\infty}\left(\mathbb{R}^{3}\right)$ function $\chi$ equal to 1 in a domain containing $\mathcal{V}(a)$ and zero in a domain containing some neighborhood $\mathcal{V}(B)$ of $B$. We set $\tilde{v}_{g^{\epsilon}}\left(\cdot, z_{p}\right):=\chi v_{g^{\epsilon}}\left(\cdot, z_{p}\right)$. For $p$ fixed, let $\left(v_{n}^{p}\right)_{n \in \mathbb{N}}$ be a sequence of Herglotz functions approximating $\tilde{v}_{g^{\epsilon}}\left(\cdot, z_{p}\right)$ in $L^{2}(\partial E)$. 
The sequence $\left(\tilde{v}_{g} \epsilon\left(\cdot, z_{p}\right)-v_{n}^{p}\right)_{(p, n) \in \mathbb{N}^{2}}$ satisfies

$\Delta\left[\tilde{v}_{g^{\epsilon}}\left(\cdot, z_{p}\right)-v_{n}^{p}\right]+k^{2}\left[\tilde{v}_{g^{\epsilon}}\left(\cdot, z_{p}\right)-v_{n}^{p}\right]=\Delta \chi v_{g_{\epsilon}}\left(\cdot, z_{p}\right)+2 \nabla \chi \cdot \nabla v_{g_{\epsilon}}\left(\cdot, z_{p}\right) \quad$ in $E$.

Since $\nabla \chi=0$ in a neighborhood of $\mathcal{V}(a)$ and in a neighborhood of $B$ and $v_{g^{\epsilon}}\left(\cdot, z_{p}\right)$ is bounded in $H^{1}(D \cap K)$, for every domain $K$ such that $K \cap \mathcal{V}(a)=\emptyset$, we deduce that the right hand side of $(5.65)$ is bounded in $L^{2}(E)$. Using the regularity of the week solution for elliptic problems with $L^{2}$ - Dirichlet boundary condition the sequence $\left(\tilde{v}_{g \epsilon}\left(\cdot, z_{p}\right)-v_{n}^{p}\right)_{p, n}$ is bounded in $L^{2}(E)$ (see [16]). Again from (5.65) and using the interior estimates, we conclude that the sequence $\left(\tilde{v}_{g \epsilon}\left(\cdot, z_{p}\right)-v_{n}^{p}\right)_{n, p}$ is bounded in $H^{2}(B)$ and in $H^{2}(D)$.

Since $\tilde{v}_{g^{\epsilon}}=0$ in $\mathcal{V}(B)$, the sequence $\left(v_{n}^{p}\right)_{n, p}$ is bounded in $H^{2}(B)$, and in particular in $L^{2}(\partial B)$. Also, since $v_{g^{\epsilon}}\left(\cdot, z_{p}\right)=\tilde{v}_{g^{\epsilon}}\left(\cdot, z_{p}\right)$ in a neighborhood of $a$, i.e. $\mathcal{V}(a)$, $\left[v_{n}^{p}-v_{g^{\epsilon}}\left(\cdot, z_{p}\right)\right]_{n, p}$ is bounded in $H^{2}(\mathcal{V}(a))$. We set $g_{R T}:=g_{n}^{p}$.

Now repeating the arguments applied for $v_{g^{\epsilon}}^{\infty}\left(\cdot, z_{p}\right)$ before replacing it by $v_{g_{R T}}^{\infty}$, we deduce that $I_{2}(B)=\infty$.

This gives a justification of the second case of Theorem 4.2.

II. Consider now the case where $\partial D \subset \bar{B}$ and assume that $a \in \partial D \cap \partial B$. We have

$$
\left\|v_{g^{\epsilon}}\left(\cdot, z_{p}\right)+\Phi\left(\cdot, z_{p}\right)\right\|_{H^{\frac{1}{2}(\partial D)}}<\epsilon .
$$

The wellposedness of the forward scattering problem gives

$$
\left\|v_{g^{\epsilon}}^{S}\left(\cdot, z_{p}\right)+\Phi\left(\cdot, z_{p}\right)\right\|_{H^{\frac{1}{2}(\partial B)}}<C \epsilon
$$

with some positive constant $C$. From this estimate we deduce that

$$
\left\|v_{g^{\epsilon}}^{S}\left(\cdot, z_{p}\right)\right\|_{H^{1}(\partial B)} \rightarrow \infty\left(z_{p} \rightarrow a\right) .
$$

This property gives the justification of the convergence of the second version of the no-response test. But as for the case I, we need to normalize it in the $L^{2}(\partial B)$-norm. In this case the argument given in I. does not work. We give another way to justify it which uses the information $\partial D \subset B$. For this, we argue as follows. We take a particular sequence $z_{p}:=a+\frac{1}{p} v(a)$, where $a \in \partial D \cap \partial B$ and $v(a)$ is the exterior unit normal at $a$ of $\partial B$. We set $z_{p}^{*}:=a-\frac{1}{p} \nu(a)$. We need the following lemma.

Lemma 5.3 There exists a constant $C>0$ such that

$$
\left\|\Phi\left(\cdot, z_{p}\right)-\Phi\left(\cdot, z_{p}^{*}\right)\right\|_{H^{\frac{1}{2}(\partial B)}} \leq C, \forall p \in \mathbb{N}
$$

Proof of Lemma 5.3 We set $\Psi_{\kappa}\left(\cdot, z_{p}\right):=\Phi_{\kappa}\left(\cdot, z_{p}\right)-\Phi_{\kappa}\left(\cdot, z_{p}^{*}\right)$. The index $\kappa$ is used to distinguish between the fundamental solution of the Helmholtz equation and the one of the Laplace equation, i.e $\kappa=0$.

The distribution $\Psi\left(\cdot, z_{p}\right):=\Psi_{\kappa}\left(\cdot, z_{p}\right)-\Psi_{0}\left(\cdot, z_{p}\right)$ satisfies

$$
\left(\Delta+\kappa^{2}\right) \Psi\left(\cdot, z_{p}\right)=\kappa^{2}\left[\Phi_{0}\left(\cdot, z_{p}^{*}\right)-\Phi_{0}\left(\cdot, z_{p}\right)\right] \text { in } \mathbb{R}^{3}
$$

where $\Phi_{0}\left(x, z_{p}\right):=\frac{1}{\left|x-z_{p}\right|}$. We take a large domain $K$ containing the sequences $\left(z_{p}\right)_{p \in \mathbb{N}}$ and $\left(z_{p}^{*}\right)_{p \in \mathbb{N}}$. Hence from $(5.68)$, we obtain that $\Psi\left(\cdot, z_{p}\right)$ is bounded in $H^{1}(K)$, because the second member of (5.68) is bounded in $L^{2}(K)$ and the boundary conditions on $\partial K$ 黑 Springer 
are bounded in $C^{m}(\partial K)$, for any $m$ in $\mathbb{N}$, which is due to the fact that the sequence $z_{p}$ is away from $\partial K$.

This implies that $\left.\Psi\right|_{\partial B}$ is bounded $H^{\frac{1}{2}}(\partial B)$. Then it is enough to prove (5.67) for $\Psi_{0}\left(\cdot, z_{p}\right)$.

We consider $\left\|\Psi_{0}\left(\cdot, z_{p}\right)\right\|_{L^{2}(\partial B)}$.

$$
\left|\frac{1}{\left|x-z_{p}\right|}-\frac{1}{\left|x-z_{p}^{*}\right|}\right|=\left|\frac{\left|x-z_{p}^{*}\right|-\left|x-z_{p}\right|}{\left|x-z_{p}\right|\left|x-z_{p}\right|}\right| \leq \frac{\left|z_{p}-z_{p}^{*}\right|}{\left|x-z_{p}\right|\left|x-z_{p}^{*}\right|} .
$$

Hence

$$
\begin{aligned}
\int_{\partial B}\left|\frac{1}{\left|x-z_{p}\right|}-\frac{1}{\left|x-z_{p}^{*}\right|}\right|^{2} d s(x) & \leq\left|z_{p}-z_{p}^{*}\right|^{2} \int_{\partial B} \frac{1}{\left|x-z_{p}\right|^{2}\left|x-z_{p}^{*}\right|^{2}} \\
& \leq C\left|z_{p}-z_{p}^{*}\right|^{2} \frac{1}{\left|z_{p}-z_{p}^{*}\right|}=C\left|z_{p}-z_{p}^{*}\right| .
\end{aligned}
$$

Then

$$
\left\|\Psi_{0}\left(\cdot, z_{p}\right)\right\|_{L^{2}(\partial B)}^{2} \leq C\left|z_{p}-z_{p}^{*}\right|
$$

with some constant $C>0$.

We consider now $\left\|\Psi_{0}\left(\cdot, z_{p}\right)\right\|_{H^{1}(\partial B)}^{2}$. It is enough to consider the estimate of the tangential derivative $\left\|\nabla_{T} \Psi_{0}\left(\cdot, z_{p}\right)\right\|_{L^{2}(\partial B)}$. We have

$$
\begin{aligned}
\nabla \Psi_{0}\left(\cdot, z_{p}\right) & =\frac{\left(x-z_{p}\right)}{\left|x-z_{p}\right|^{3}}-\frac{\left(x-z_{p}^{*}\right)}{\left|x-z_{p}^{*}\right|^{3}}=\frac{\left(x-z_{p}\right)}{\left|x-z_{p}\right|^{3}}+\frac{\left(z_{p}-z_{p}^{*}\right)}{\left|x-z_{p}\right|^{3}}-\frac{\left(x-z_{p}^{*}\right)}{\left|x-z_{p}^{*}\right|^{3}} \\
& =\frac{\left(z_{p}-z_{p}^{*}\right)}{\left|x-z_{p}\right|^{3}}+\left(x-z_{p}^{*}\right)\left[\frac{\left|x-z_{p}^{*}\right|^{3}-\left|x-z_{p}\right|^{3}}{\left|x-z_{p}^{*}\right|^{3}\left|x-z_{p}\right|^{3}}\right] .
\end{aligned}
$$

Hence the tangential derivative satisfies

$$
\left|\nabla_{T} \Psi_{0}\left(\cdot, z_{p}\right)\right| \leq \frac{\left|z_{p}-z_{p}^{*}\right|}{\left|x-z_{p}\right|^{3}}+\left|x-z_{p}^{*}\right| \frac{C_{1}\left|z_{p}-z_{p}^{*}\right|}{\left|x-z_{p}^{*}\right|^{2}\left|x-z_{p}\right|^{3}}
$$

where $c_{1}>0$ is a constant. Then, the square integrals of the first and the second terms of the left hand side of the last inequality imply

$$
\int_{\partial B}\left|\nabla \Psi_{0}\left(\cdot, z_{p}\right)\right|^{2} d s(x) \leq \frac{C_{2}}{\left|z_{p}-z_{p}^{*}\right|}
$$

which means that

$$
\left\|\Psi_{0}\left(\cdot, z_{p}\right)\right\|_{H^{1}(\partial B)}^{2} \leq \frac{C_{3}}{\left|z_{p}-z_{p}^{*}\right|}
$$

with some positive constants $C_{2}, C_{3}$. From (5.69) and (5.70) and the interpolation theorem between $L^{2}(\partial B):=H^{0}(\partial B)$ and $H^{1}(\partial B)$, we get

$$
\left\|\Psi_{0}\left(\cdot, z_{p}\right)\right\|_{H^{\frac{1}{2}(\partial B)}} \leq C_{4}
$$

with some positive constant $C_{4}$. This ends the proof of Lemma 5.67. 
From (5.67) and (5.66), we deduce that

$$
\left\|v_{g^{\epsilon}}^{S}\left(\cdot, z_{p}\right)+\Phi\left(\cdot, z_{p}^{*}\right)\right\|_{H^{\frac{1}{2}(\partial B)}} \leq C_{5}
$$

for some positive constant $C_{5}$.

For every $p$ fixed, let $v_{g_{n}}$ be a Herglotz sequence of functions tending to $v_{g^{\epsilon}}^{s}\left(\cdot, z_{p}\right)$ in $H^{\frac{1}{2}}(\partial B)$, i.e.

$$
\forall p \in \mathbb{N}, \exists N(p) / \forall n \geq N(p) \quad \text { we have }\left\|v_{g_{n}^{p}}-v_{g^{\epsilon}}^{S}\left(\cdot, z_{p}\right)\right\|_{H^{\frac{1}{2}}(\partial B)} \leq \epsilon
$$

From (5.67) and (5.72), we have

$$
\forall p \in \mathbb{N}, \exists N(p) / \forall n \geq N(p) \quad \text { we have }\left\|v_{g_{n}^{p}}-\Phi\left(\cdot, z_{p}^{*}\right)\right\|_{H^{\frac{1}{2}(\partial B)}} \leq C_{6} .
$$

Hence, from $\partial D \subset B$ and by the wellposedness of the scattering problem we obtain

$$
\left\|v_{g_{n}^{p}}^{s}-\Phi^{S}\left(\cdot, z_{p}^{*}\right)\right\|_{H^{\frac{1}{2}(\partial B)}} \leq C_{7}
$$

where $C_{6}, C_{7}>0$ are constants. From (5.73), we have $\left\|v_{g_{n}^{p}}\right\|_{L^{2}(\partial B)}^{2} \leq C_{8} \ln p$ for $p$ large enough and $C_{8}>0$ is a constant. By setting $\beta_{p}:=\left\|v_{g_{n}}^{p}\right\|_{L^{2}(\partial B)}^{-1}$ we have

$$
\left\|\beta_{p} v_{g_{n}^{p}}^{s}\left(\cdot, z_{p}\right)\right\|_{H^{\frac{1}{2}(\partial B)}}^{2} \geq \frac{\left\|\Phi^{s}\left(\cdot, z_{p}^{*}\right)\right\|^{2} H^{\frac{1}{2}(\partial B)}}{C_{8} \ln p}-\frac{C_{7}^{2}}{C_{8}}(\ln p)^{-1} .
$$

Since $\left\|\Phi^{S}\left(\cdot, z_{p}^{*}\right)\right\|_{H^{\frac{1}{2}(\partial B)}} \geq C_{9} d\left(z_{p}^{*}, \partial B\right)=C_{9} \frac{1}{p}$, we deduce that

$$
\left\|\beta_{p} v_{g_{N(p)}^{p}}^{s}\left(\cdot, z_{p}\right)\right\|_{H^{\frac{1}{2}}(\partial B)} \rightarrow \infty .
$$

Hence, $v_{g r t}:=\beta_{p} v_{g_{N(p)}^{p}}$ satisfies

$$
\left\|v_{g_{R T}}\right\|_{L^{2}(\partial B)}=1 \text { and }\left\|v_{g_{R T}}^{s}\right\|_{H^{1}(\partial B)} \rightarrow \infty .
$$

Arguing as in the part I, taking $v_{g_{R T}}^{\infty}$ instead of $v_{g^{\epsilon}\left(\cdot, z_{p}\right)}^{\infty}$, we deduce that $I_{2}(B)=\infty$.

Since the case one is always justified as the forward problem is well posed, we deduce the convergence of the second version of the no-response test.

\section{Equivalence of the two versions}

We will show that with respect to the convergence properties the two versions are equivalent. Precisely, we show how the singular sequences creating the blowup for the corresponding indicator functions are linked.

Theorem 6.1 Consider the formulas (2.13) and (4.41) of the no-response test. Then the convergence of the first version implies the convergence of the second version.

Proof According to our assumption, the first version is convergent, i.e. we have $\bar{D} \subset B$ implies $I_{1}(B)=0$ and $\bar{D} \not \subset B$ implies $I_{1}(B)=\infty$. We will show that the same implications hold for the indicator function $I_{2}$. 
For the case $\bar{D} \subset B$, we have $I_{1}(B)=I_{2}(B)=0$ which is justified by the wellposedness of the direct problem. We will show that for $\bar{D} \not \subset B$, we obtain the logical implication

$$
\left(I_{1}(B)=\infty\right) \Longrightarrow\left(I_{2}(B)=\infty\right),
$$

which implies the above theorem.

We suppose that $I_{1}(B)=\infty$. Let $\left(f_{n}, g_{n}\right)$ be a sequence in $L^{2}(\mathbb{S}) \times L^{2}(\mathbb{S})$ such that $\left\|v_{f_{n}}\right\|_{L^{2}(\partial B)}<\frac{1}{n},\left\|v_{g_{n}}\right\|_{L^{2}(\partial B)}<\frac{1}{n}$ and

$$
\lim _{n \rightarrow \infty} \int_{\mathbb{S}^{2}} u^{\infty}(-\theta, d) f_{n}(\theta) g_{n}(d) \mathrm{d} s(\theta) \mathrm{d} s(d)=\infty .
$$

We will investigate the functions $v_{g_{n}}^{i}$ and its far field $v_{g_{n}}^{\infty}$. To this end consider the solvability of the integral Eq. (4.38) with $v_{g}^{\infty}$ on the right-hand side as described in (4.41). As in Sect. 3, we distinguish two cases.

1. For some $v_{g_{n_{0}}}^{\infty}$ the equation (4.38) is not solvable. In this case we deduce that $I_{2}(B)=\infty$.

2. For every $v_{g_{n}}^{\infty},(4.38)$ is solvable. In this case Lemma 4.4 implies

$$
v_{g_{n}}^{s}(x)=\int_{\partial B} \Phi(x, y) \psi_{n}(y) \mathrm{d} s(y), \quad x \in \mathbb{R}^{3} \backslash(B \cup D),
$$

hence the far field patterns enjoy the property

$$
v_{g_{n}}^{\infty}(\theta)=\frac{1}{4 \pi} \int_{\partial B} \psi_{n}(y) \mathrm{e}^{-i \kappa \theta \cdot y} \mathrm{~d} s(y) .
$$

Now multiplying the last equality by $f_{n}$ and integrating over $\mathbb{S}$, we obtain

$$
\int_{\mathbb{S}} v_{g_{n}}^{\infty}(-\theta) f_{n}(\theta) \mathrm{d} s(\theta)=\frac{1}{4 \pi} \int_{\partial B} \psi_{n}(y) v_{f_{n}}(y) \mathrm{d} s(y) .
$$

If $\psi_{n}$ is bounded in $L^{2}(\partial B)$, then $\int_{\partial B} \psi_{n}(y) v_{f_{n}}(y) \mathrm{d} s(y)$ is also bounded. Hence

$$
\int_{\mathbb{S}} \int_{\mathbb{S}} u^{\infty}(-\theta, d) f_{n}(\theta) g_{n}(d) \mathrm{d} s(\theta) \mathrm{d} s(d)=\int_{\mathbb{S}} v_{g_{n}}^{\infty}(-\theta) f_{n}(\theta) \mathrm{d} s(\theta)
$$

is also bounded. This contradicts (6.74) and hence we deduce that the sequence $\left\|\psi_{n}\right\|_{L^{2}(\partial B)}$ is unbounded. Therefore, we have proven that $I_{2}(B)=\infty$.

6.1 The second version implies the first version

Theorem 6.2 Consider the formulas (2.13) and (4.41) of the no-response test. Then the convergence of the second version implies the convergence of the first version.

Proof Suppose that the second version is convergent, i.e. we have $\bar{D} \subset B$ implies $I_{2}(B)=0$ and $\bar{D} \not \subset B$ implies $I_{2}(B)=\infty$. We will show that the same implications hold for the indicator function $I_{1}$. 
The case $\bar{D} \subset B$ can be handled as in the proof of Theorem 6.1. We suppose that $\bar{D} \not \subset B, B$ is a non-vibrating domain and $I_{2}(B)=\infty$. Our goal is to show that under these assumptions $I_{1}(B)=\infty$.

Since $I_{2}(B)=\infty$, there exists a sequence $\left(\psi_{n}^{\alpha}\right) \subset L^{2}(\partial B)$ of regularized solution of (4.38) with right-side $v_{g_{n}}^{\infty}$ such that $\left\|v_{g_{n}}^{i}\right\|_{L^{2}(\partial B)}<\frac{1}{n}$ and

$$
\limsup _{n \rightarrow \infty} \lim _{\alpha \rightarrow 0}\left\|\psi_{n}^{\alpha}\right\|_{L^{2}(\partial B)}=\infty \text {. }
$$

We split the proof into two parts which investigate the following cases.

1. For every $n$ in $\mathbb{N}$, the integral Eq. (4.38) with right-side $v_{g_{n}}^{\infty}$ is solvable. In this case, according to Lemma 4.3, we pass to the limit $\alpha \rightarrow 0$ to obtain

$$
\limsup _{n \rightarrow \infty}\left\|\psi_{n}\right\|_{L^{2}(\partial B)}=\infty
$$

where $\psi_{n}$ is the sequence of the solutions of (4.38) with right-hand side $v_{g_{n}}^{\infty}$.

2. For some $n_{0}$, we have $\lim _{\alpha \rightarrow 0}\left\|\psi_{n_{0}}^{\alpha}\right\|_{L^{2}(\partial B)}=\infty$, i.e the integral Eq. (4.38) with right-side $v_{g_{n_{0}}}^{\infty}$ is not solvable.

First case We multiply the equation

$$
S^{\infty} \psi_{n}=v_{g_{n}}^{\infty}
$$

by $f \in L^{2}(\mathbb{S})$ and integrate over $\mathbb{S}$ to obtain

$$
\int_{\mathbb{S}} S^{\infty} \psi_{n}(-\theta) f(\theta) \mathrm{d} s(\theta)=\int_{\mathbb{S}} v_{g_{n}}^{\infty}(-\theta) f(\theta) \mathrm{d} s(\theta) .
$$

The left-hand side becomes

$$
\begin{aligned}
\int_{\mathbb{S}} S^{\infty} \psi_{n}(-\theta) f(\theta) \mathrm{d} s(\theta) & =\int_{\mathbb{S}} \int_{\partial B} \mathrm{e}^{i k \theta \cdot x} \psi_{n}(x) \mathrm{d} s(x) f(\theta) \mathrm{d} s(\theta) \\
& =\int_{\partial B} \int_{\mathbb{S}} \mathrm{e}^{i k \theta \cdot x} f(\theta) \mathrm{d} s(\theta) \psi_{n}(x) \mathrm{d} s(x) \\
& =\int_{\partial B} v_{f}(x) \psi_{n}(x) \mathrm{d} s(x) .
\end{aligned}
$$

For every $n$ fixed, by the denseness of the range of the Herglotz operator in $L^{2}(\partial B)$, we take $\left(v_{f_{n}^{p}}\right)_{p \in \mathbb{N}}$ such that

$$
v_{f_{n}^{p}} \longrightarrow \frac{\epsilon}{2} \frac{\overline{\psi_{n}}}{\left\|\psi_{n}\right\|_{L^{2}(\partial B)}}, \quad p \rightarrow \infty,
$$

in $L^{2}(\partial B)$. Hence, for $n$ fixed, there exists $N_{0}(n)$, such that $\forall p>N_{0}(n)$,

$$
\left\|v_{f_{n}^{p}}\right\|_{L^{2}(\partial B)}<\epsilon .
$$


Then, for every $n$ fixed, we have

$$
\begin{aligned}
\lim _{p \rightarrow \infty} \int_{\mathbb{S}} S^{\infty} \psi_{n}(\theta) f_{p}^{n}(\theta) \mathrm{d} s(\theta) & =\lim _{p \rightarrow \infty} \int_{\partial B} v_{f_{p}^{n}}(x) \psi_{n}(x) \mathrm{d} s(x) \\
& =\int_{\partial B} \frac{\epsilon}{2} \frac{\left|\psi_{n}\right|^{2}(x)}{\left\|\psi_{n}\right\|_{L^{2}(\partial B)}} \mathrm{d} s(x) \\
& =\frac{\epsilon}{2}\left\|\psi_{n}\right\|_{L^{2}(\partial B)} .
\end{aligned}
$$

Hence

$$
\limsup _{n \rightarrow \infty} \lim _{p \rightarrow \infty} \int_{\mathbb{S}} v_{g_{n}}^{\infty}(\theta) f_{p}^{n}(\theta) \mathrm{d} s(\theta)=\infty .
$$

The right-hand side of (6.76) with $f=f_{p}^{n}$ such that (6.79) is satisfied is a lower bound for $I_{1}(B)$. Now, from $(6.81)$ we obtain $I_{1}(B)=\infty$.

Second case Since $v_{g_{n_{0}}}^{\infty}$ is not in the range of $S^{\infty}$, then $v_{g_{n_{0}}}^{s}$ cannot be analytically extended up to $\mathbb{R}^{3} \backslash B$. Indeed, if it is analytically extendable up to $\mathbb{R}^{3} \backslash B$, then it satisfies $\Delta v_{g_{n_{0}}}^{s}+k^{2} v_{g_{n_{0}}}^{s}=0$ in a neighborhood of $\mathbb{R}^{3} \backslash B$. In particular $\left.v_{g_{n_{0}}}^{s}\right|_{\partial B} \in H^{1}(\partial B)$. Then, the equation $S \psi=\left.v_{g_{n_{0}}}^{s}\right|_{\partial B}$ is solvable in $L^{2}(\partial B)$ and then $S^{\infty} \psi=v_{g_{n_{0}}}^{\infty}$, which is a contradiction.

We need the following theorem which gives a useful geometrical property related to the notion of homotopy. Let $\Gamma$ be an open ball of $\mathbb{R}^{3}$. A bounded domain whose boundary is $C^{m}$ diffeomorphic to a unit sphere is called a $C^{m}$ spherical domain. In the following theorem we take $m=2$.

Theorem 6.3 Let $q \in \partial B$. Then, there is a $C^{2}$ spherical domain $B(q)$ satisfying the following properties (i) and (ii).

1. $B \subset B(q) \subset \Gamma$ and $q \in \partial B(q)$.

2. There exists a family of $C^{2}$ spherical domains $B_{t}(q)(0 \leq t \leq 1)$ such that $B_{0}(q)=$ $B(q), B_{1}(q)=\Gamma, \overline{B_{t}(q)} \subset B_{t^{\prime}}(q)\left(t<t^{\prime}\right)$ and the dependency of $B_{t}$ on $t$ is $C^{2}$.

The proof of this theorem will be given in the appendix. We have the following lemma.

Lemma 6.4 There exists $x_{0}$ in $\partial B$ and $B_{t_{0}}\left(x_{0}\right), t_{0}>0$, such that $v_{g_{n_{0}}}^{s}$ is extendable analytically up to $\mathbb{R}^{3} \backslash B_{t}\left(x_{0}\right), t>t_{0}$, but not up to $\mathbb{R}^{3} \backslash B_{t_{0}}\left(x_{0}\right)$.

Proof of Lemma 6.4 Suppose that for every $x \in \partial B, v_{g_{n_{0}}}^{t}$ is extendable analytically up to $\mathbb{R}^{3} \backslash B_{t}(x), \forall t \in[0,1]$. Hence this would mean that $v_{g_{n_{0}}}^{s}$ is extendable analytically near any point of $\mathbb{R}^{3} \backslash B$. This is impossible.

From Lemma 6.4, there exist $x_{0} \in \partial B$ and $B_{t_{0}}\left(x_{0}\right), t_{0}>0$, such that $v_{g_{n_{0}}}^{s}$ is extendable analytically up to $\mathbb{R}^{3} \backslash B_{t}\left(x_{0}\right), t>t_{0}$, but not up to $\mathbb{R}^{3} \backslash B_{t_{0}}\left(x_{0}\right)$. We set $B_{t_{0}}:=$ $B_{t_{0}}\left(x_{0}\right)$. Hence there exists $z_{0} \in \partial B_{t_{0}}$ and an open neighborhood $N\left(z_{0}\right)$ of $z_{0}$ such that $\overline{N\left(z_{0}\right)} \cap \bar{B}=\emptyset$ and $v_{g_{n_{0}}}^{s}$ is not analytically extendable into any open subset of $N\left(z_{0}\right)$.

Lemma 6.5 Let $z_{p}$ be a sequence of points in $\mathbb{R}^{3} \backslash B_{t_{0}}$ such that $z_{p}$ tends to $z_{0}$. If for some positive $\rho \in \mathbb{R}$ the set

$$
\left\{\sup _{|h|=1} \rho^{\mu} \frac{\left|(h \cdot \nabla)^{\mu} v_{g_{n_{0}}}^{s}\left(z_{p}\right)\right|}{\mu !}, \quad \mu \in \mathbb{Z}_{+}\right\}
$$


is uniformly bounded by a constant $c>0$, then $v$ can be analytically extended near the point $z_{o}$.

Proof of Lemma 6.5 From the boundedness of (6.82) we derive that the series

$$
\sum_{\mu \in \mathbb{Z}_{+}} \frac{1}{\mu !}((x-z) \cdot \nabla)^{\mu} v_{g_{n_{0}}}^{s}(z)
$$

has a majorant $\sum_{\mu \in \mathbb{Z}_{+}} c\left|\frac{x-z_{p}}{\rho}\right|^{\mu}$ and hence it is absolutely converging for $x \in B\left(z_{p}, \rho\right)$ and for all the points $z_{p}$. Taking $z_{p}$ close enough to $z_{0}$, we deduce that $v$ is analytically extendable into some open neighborhood of $z_{0}$.

As a consequence of the preceding lemma, for any sequence $z_{p}$ of points in $\mathbb{R}^{3} \backslash B_{t_{0}}$ tending to $z_{0}$, there exist a sequence $h_{p} \in \mathbb{S}, \mu_{p} \in \mathbb{N}$ such that

$$
\lim _{p \rightarrow \infty} \rho^{\mu_{p}} \frac{\left|\left(h_{p} \cdot \nabla\right)^{\mu_{p}} v_{g_{n_{0}}}^{s}\left(z_{p}\right)\right|}{\mu_{p} !}=\infty .
$$

Let $\epsilon>0$ be fixed. We set

$$
\psi_{p}(x):=\frac{\epsilon}{2 \beta\left(z_{p}, \mu\right)}\left(h_{p} \cdot \nabla_{z}\right)^{\mu_{p}} \Phi\left(x, z_{p}\right),
$$

where

$$
\beta(p):=\sup _{y \in \bar{B}}\left\{\left|\left(h_{p} \cdot \nabla_{z}\right)^{\mu_{p}} \Phi\left(y, z_{p}\right)\right|\right\}
$$

is introduced for normalization.

Let $p$ be fixed. Then, we can find a sequence of densities $g_{n}^{p} \in L^{2}(\mathbb{S})$ such that $v_{g_{n}^{p}}$ tends to $\psi_{p}$ in $L^{2}\left(E_{z_{p}}\right)$ for some non-vibrating domain $E_{z_{p}}$ such that $\overline{B_{t_{0}}} \subset E_{z_{p}}$, $z_{p} \notin E_{z_{p}}$ and $\mathbb{R}^{3} \backslash \overline{E_{z_{p}}}$. By the interior regularity, $v_{g_{n}}$ tends to $\psi_{p}$ in $C^{1}\left(\overline{B_{t_{0}}}\right)$.

Since

$$
\left\|\psi_{p}\right\|_{C(\bar{B})} \leq \frac{\epsilon}{2}
$$

then

$$
\left\|v_{g_{n}}\right\|_{C(\bar{B})}<\epsilon
$$

for $n$ large enough. Now from the identity (2.8) applied on $\mathbb{R}^{3} \backslash \overline{B_{s_{0}}}$ for $v_{g_{n_{0}}}^{s}$ and $e^{-i k \theta \cdot x}$, we have:

$$
\int_{\mathbb{S}^{2}} u^{\infty}(-\theta, d) g_{n_{0}}(d) g_{n}^{p}(\theta) \mathrm{d} s(\theta) \mathrm{d} s(d)=\frac{1}{4 \pi} \int_{\partial B_{t_{0}}}\left\{\frac{\partial v_{g_{n_{0}}}^{s}}{\partial v} v_{g_{n}}-\frac{\partial v_{g_{n}}^{p}}{\partial v} v_{g_{n_{0}}}^{s}\right\} \mathrm{d} s(x)
$$

Taking the limit with respect to $n$, we have:

$$
\begin{gathered}
\lim _{n \rightarrow \infty} \int_{\mathbb{S}^{2}} u^{\infty}(-\theta, d) g_{n_{0}}(d) g_{n}^{p}(\theta) \mathrm{d} s(\theta) \mathrm{d} s(d) \\
=\frac{1}{4 \pi} \int_{\partial B_{t_{0}}}\left\{\frac{\partial v_{g_{n_{0}}}^{s}}{\partial v} \psi_{p}-\frac{\partial \psi_{p}}{\partial v} v_{g_{n_{0}}}^{s}\right\} \mathrm{d} s(x)
\end{gathered}
$$


Let $\Omega_{R}$ be an open ball with radius centered at the origin such that $\overline{B_{t_{0}}} \subset \Omega_{R}$. Applying the Green's formula in $\Omega_{R} \backslash \bar{D}$ for $v_{g_{n_{0}}}^{s}$ and $\psi_{p}$, we deduce that

$$
\begin{aligned}
& \int_{\partial B_{t_{0}}}\left\{\frac{\partial v_{g_{n_{0}}}^{s}}{\partial v} \psi_{p}-\frac{\partial \psi_{p}}{\partial v} v_{g_{n_{0}}}^{s}\right\} \mathrm{d} s(x) \\
& =\frac{\epsilon}{2 \beta(p)}\left(h_{p} \cdot \nabla_{z}\right)^{\mu_{p}} v_{g_{n_{0}}}^{s}\left(z_{p}\right)-\int_{\partial \Omega_{R}}\left\{\frac{\partial v_{g_{n_{0}}}^{s}}{\partial v} \psi_{p}-\frac{\partial \psi_{p}}{\partial v} v_{g_{n_{0}}}^{s}\right\} \mathrm{d} s(x) \\
& =\frac{\epsilon}{2 \beta(p)}\left(h_{p} \cdot \nabla_{z}\right)^{\mu_{p}} v_{g_{n_{0}}}^{s}\left(z_{p}\right)-\int_{\partial \Omega_{R}}\left\{\left(\frac{\partial v_{g_{n_{0}}}^{s}}{\partial v}-i \kappa v_{g_{n_{0}}}^{s}\right) \psi_{p}-\left(\frac{\partial \psi_{p}}{\partial v}-i \kappa \psi_{p}\right) v_{g_{n_{0}}}^{s}\right\} \mathrm{d} s(x) .
\end{aligned}
$$

It is easy to see that

$$
\psi_{p}(x)=\frac{\epsilon}{8 \pi \beta_{p}}\left(\frac{-i \kappa h_{p} \cdot\left(x-z_{p}\right)}{\left|x-z_{p}\right|}\right)^{\left|\mu_{p}\right|} \frac{\mathrm{e}^{i \kappa\left|x-z_{p}\right|}}{\left|x-z_{p}\right|}+O\left(\frac{1}{|x|^{2}}\right) \text { for }|x| \rightarrow \infty .
$$

By

$$
\frac{\mathrm{d}}{\mathrm{d} r} h_{p} \cdot\left(x-z_{p}\right)=\frac{x \cdot h_{p}}{|x|}
$$

and

$$
\frac{\mathrm{d}}{\mathrm{d} r}\left|x-z_{p}\right|=1+O\left(\frac{1}{|x|}\right),\left|x-z_{p}\right|=|x|-\frac{x}{|x|} \cdot z_{p}+O\left(\frac{1}{|x|}\right)
$$

where $r:=|x| \rightarrow \infty$, we have

$$
\frac{\mathrm{d}}{\mathrm{d} r} \psi_{p}(x)-i \kappa \psi_{p}(x)=O\left(\frac{1}{|x|^{2}}\right), \quad(|x| \rightarrow \infty) .
$$

From its definition, $v_{g_{n_{0}}}^{s}$ satisfies

$$
v_{g_{n_{0}}}^{s}=O\left(\frac{1}{|x|}\right) \quad \text { and } \quad \frac{\mathrm{d}}{\mathrm{d} r} v_{g_{n_{0}}}^{s}(x)-i \kappa v_{g_{n_{0}}}^{s}=O\left(\frac{1}{|x|^{2}}\right), \quad(|x| \rightarrow \infty) .
$$

From (6.87) and (6.88), the second term of the right hand side of (6.86) tends to zero as $R$ tends to $\infty$. Hence, we get

$$
\int_{\partial B_{t_{0}}}\left\{\frac{\partial v_{g_{n_{0}}}^{s}}{\partial v} \psi_{p}-\frac{\partial \psi_{p}}{\partial v} v_{g_{n_{0}}}^{s}\right\} \mathrm{d} s(x)=\frac{\epsilon}{2 \beta(p)}\left(h_{p} \cdot \nabla_{z}\right)^{\mu_{p}} v_{g_{n_{0}}}^{s}\left(z_{p}\right)
$$

Next, in order to analyze the behavior of the right hand side of (6.89), we have to estimate $\beta(p)$.

To begin with, we use $\hat{x}=\left(\hat{x}_{1}, \hat{x}_{2}, \hat{x}_{3}\right) \in \mathbb{C}^{3}$ for complexifying $x=\left(x_{1}, x_{2}, x_{3}\right) \in \mathbb{R}^{3}$ such that the real part of $\hat{x}_{j}$ is $\operatorname{Re}\left(\hat{x}_{j}\right)=x_{j}$.

Since $\bar{B} \subset B_{t_{0}}$, then the distance $d\left(z_{0}, B\right)$ is positive. Then for any $x \in \bar{B}$, there exist an open neighborhood of $U_{x} \subset \mathbb{R}^{3}$ of $x$ and complex open polydiscs $V_{z_{0}} \subset \mathbb{C}^{3}$ 
centered $z_{0}$ such that

$$
\left\{\begin{array}{l}
U_{x} \cap V_{z_{0}}=\varnothing \text { and } \\
(y-\hat{z}) \cdot(y-\hat{z}):=\sum_{j=i}^{3}\left(\hat{y}_{j}-\hat{z}_{j}\right)^{2} \neq 0 \quad \text { for any } y \in U_{x} \quad \text { and } \quad \hat{z} \in V_{z}
\end{array}\right.
$$

By the compactness of $\bar{B}$ in $\mathbb{R}^{3}$, there exist $x^{(k)} \in \bar{B}(1 \leq k \leq K)$ such that $\bar{B} \subset$ $\cup_{k=1}^{K} U_{x^{(k)}}$. Let $V_{z_{0}}^{(k)}$ be the polydisc centered at $z_{0}$ associated with $U_{x^{(k)}}$ satisfying (6.90).

Now, we define a complex open polydisc $V$ centered at $z_{0}$ by $V:=\cap_{k=1}^{K} V_{z_{0}}^{(k)}$. Then, we have

$$
\left\{\begin{array}{l}
U_{x^{(k)}} \cap V=\emptyset(1 \leq k \leq K) \text { and } \\
(x-\hat{z}) \cdot(x-\hat{z}) \neq 0 \text { for any } x \in U_{x^{(k)}} \quad \text { and } \quad \hat{z} \in V ; 1 \leq k \leq K .
\end{array}\right.
$$

Moreover, by considering large $p$, we can assume that $z_{p} \in V$.

For each $k(1 \leq k \leq K), \Phi(x, z)$ has a natural analytic extension $\tilde{\Phi}(x, \hat{z})$ :

$$
\hat{\Phi}(x, \hat{z})=\frac{\mathrm{e}^{i \kappa\{(x-\hat{z}) \cdot(x-\hat{z})\}^{\frac{1}{2}}}}{4 \pi\{(x-\hat{z}) \cdot(x-\hat{z})\}^{\frac{1}{2}}} \quad \text { for } x \in U_{x^{(k)}} \quad \text { and } \quad \hat{z} \in V,
$$

where we take the branch of $\{(x-\hat{z}) \cdot(x-\hat{z})\}^{\frac{1}{2}}$ such that it is equal to $|x-z|$ when $\hat{z}=z$.

By the Cauchy integral formula, there exist $C_{k}>0$ and $\rho_{k}>0$ such that

$$
\left|\left(h_{p} \cdot \nabla_{z}\right)^{\mu_{p}} \Phi\left(x, z_{p}\right)\right| \leq C_{k} \frac{\mu_{p} !}{\rho_{k}^{\mu_{p}}}, \quad \text { for } x \in U_{x^{(k)}}, p \in \mathbb{N} .
$$

Hence by taking

$$
\rho:=\min _{1 \leq k \leq K} \rho_{k} \quad \text { and } \quad C:=\max _{1 \leq k \leq K} C_{k}
$$

we have

$$
\left|\left(h_{p} \cdot \nabla_{z}\right)^{\mu_{p}} \Phi\left(x, z_{p}\right)\right| \leq C \frac{\mu_{p} !}{\rho^{\mu_{p}}} \quad \text { for } x \in \bar{B} \quad \text { and } p \in \mathbb{N} .
$$

By (6.94), the right hand side of (6.89) is estimated from below by

$$
\frac{\epsilon}{2 C} \frac{\rho^{\mu_{p}}}{\mu_{p} !}\left|\left(h_{p} \cdot \nabla_{z}\right)^{\left(\mu_{p}\right)} v_{g_{n_{0}}}\left(z_{p}\right)\right| .
$$

This last sequence is unbounded by (6.84).

Finally from (6.85), we deduce that there exists a subsequence $v_{g_{n}^{p}}$ such that:

$$
\lim _{p \rightarrow \infty} \lim _{n \rightarrow \infty} \int_{\mathbb{S}^{2}} u^{\infty}(-\theta, d) v_{g_{n}}(d) v_{g_{n}^{p}}(\theta) \mathrm{d} s(\theta) \mathrm{d} s(d)=\infty,
$$

i.e. $I_{1}(B)=\infty$.

To finish the proof, let us show how we get (6.93). Indeed, from the Cauchy formula, we represent $\hat{\Phi}(x, \hat{z})$ by

$$
\hat{\Phi}(x, \hat{z})=\frac{1}{(2 \pi i)^{3}} \int_{\Gamma} \frac{\hat{\Phi}(x, \zeta)}{\left(\zeta_{1}-\hat{z}_{1}\right) \cdot\left(\zeta_{2}-\hat{z}_{2}\right) \cdot\left(\zeta_{3}-\hat{z}_{3}\right)} \mathrm{d} \zeta
$$


where $\Gamma:=\left\{\zeta=\left(\zeta_{1}, \zeta_{2}, \zeta_{3}\right) ;\left|\zeta_{j}-\left(z_{0}\right)_{j}\right|=r\right.$ for $\left.1 \leq j \leq 3\right\}$ such that $[\Gamma]:=\{\hat{z}$ : $\left|\hat{z}_{j}-\left(z_{0}\right)_{j}\right| \leq r$ for $\left.1 \leq j \leq 3\right\} \subset V$. We set $D(\rho):=\left\{\hat{z}:\left|\hat{z}_{j}-\left(z_{0}\right)_{j}\right| \leq \rho\right.$ for $\left.1 \leq j \leq 3\right\}$ with $\rho<r$. Since

$$
\left|\zeta_{j}-\hat{z}_{j}\right| \geq\left|\zeta_{j}-\left(z_{o}\right)_{j}\right|-\left|\hat{z}_{j}-\left(z_{o}\right)_{j}\right| \geq r-\rho
$$

and

$$
|\hat{\Phi}(x, \zeta)| \leq M, \quad \text { for } x \in U_{x^{(k)}}, \zeta \in V
$$

with some positive constant $M$, then we have

$$
\left|\partial_{\hat{z}}^{\alpha} \hat{\Phi}(x, \hat{z})\right| \leq \frac{M \alpha ! \rho^{3}}{(r-\rho)^{|\alpha|}+3}, \quad \text { for } x \in U_{x^{(k)}}, \quad \hat{z} \in D(\rho)
$$

Hence

$$
\left|\partial_{\hat{z}}^{\alpha} \hat{\Phi}(x, \hat{z})\right| \leq \frac{M \alpha !}{\left(\frac{r}{2}\right)^{|\alpha|}}, \quad \text { for } x \in U_{x^{(k)}}, \hat{z} \in D\left(\frac{r}{2}\right) .
$$

Acknowledgments During the preparation of this work, the forth author was supported by Japan Society for Promotion of Sciences and the Institute for Numerical and Applied Mathematics of Göttingen University. He thanks these two institutions for these supports and other facilities. The authors would like to express their sincere acknowledgments to the referee for his remarks and suggestions.

\section{Appendix: Proof of Theorem 6.3}

In this appendix we recall the content of Theorem 6.3 and give its proof. Although all the argument which we are going to give here does not depend on the space dimension, we will confined to the 3 space dimension. Let $\Gamma \subset \mathbb{R}^{3}$ be an open ball and $\Delta$ be a domain with the $C^{m}(m \in\{\mathbb{N}, \infty\})$ smooth boundary. We assume that $\bar{\Delta} \subset \Gamma$ and $\bar{\Gamma} \backslash \Delta$ is connected. A bounded domain whose boundary is $C^{m}$ diffeomorphic to a unit sphere is called $C^{m}$ spherical domain. For this part of the paper, we abuse using the notation $\Delta$ which has not to be confused with the symbol of the Laplace operator.

Theorem 7.6 Let $q \in \partial \Delta$. Then, there is a $C^{m}$ spherical domain $B(q)$ satisfying the following properties (1) and (2).

1. $\Delta \subset B(q) \subset \Gamma$ and $q \in \partial B(q)$.

2. There exists a family of $C^{m}$ spherical domain $B_{s}(q)(0 \leq s \leq 1)$ such that $B_{0}(q)=$ $B(q), B_{1}(q)=\Gamma, \overline{B_{s}(q)} \subset B_{s^{\prime}}(q)\left(s<s^{\prime}\right)$ and the dependency of $B_{s}(q)$ on $s$ is $C^{m}$. We call such a family $\left\{B_{s}(q)\right\}$ strict deformation family of $q$ and $\Gamma$.

Proof Take $p \in \partial \Gamma$. Since $\bar{\Gamma} \backslash \Delta$ is connected, we can take a $C^{m+1}$ curve $\ell:=\{\ell(t) ; 0 \leq$ $t \leq 1\}$ such that $\ell(0)=q, \ell(1)=p$ and $\ell(t) \in \Gamma \backslash \bar{\Delta}$ for any $t \in(0,1)$. Moreover we may assume $\ell$ has no self-intersecting points. Extend $\ell$ a little bit beyond $p$ and $q$. So, we consider that $\ell$ is defined by $\ell:=\{\ell(t) ;-\varepsilon<t<1+\varepsilon\}$ with small $\varepsilon>0$. Also, we can assume that $\ell$ is transversal to $\partial \Delta$ and $\partial \Gamma$.

Now, we consider a subbundle $E$ of the tangent bundle $T \mathbb{R}^{3}$ over $\ell$ with fiber $\left\{\frac{d \ell}{d t}(t)\right\}^{\perp} \subset \mathbb{R}^{3}$ at $\ell(t)$. Since $\ell$ is retractable, $E$ is a trivial bundle. Hence, there exist linearly independent sections $v_{j}(t) \in C^{m}((-\varepsilon, 1+\varepsilon), E)(j=1,2)$ such that a tubular 
neighborhood T of $\ell$ is given by $T=\left\{\ell(t)+x v_{1}(t)+y v_{2}(t) ; t \in(-\varepsilon, 1+\varepsilon),(x, y) \in V\right\}$ with an open neighborhood $V \subset \mathbb{R}^{2}$ of $(0,0) \in \mathbb{R}^{2}$. This representation of $T$ gives a $C^{m}$ diffeomorphism $h$ :

$$
h: U \ni(t, x, y) \longrightarrow \ell(t)+x v_{1}(t)+y v_{2}(t) \in T,
$$

where $U:=(-\varepsilon, 1+\varepsilon) \times V$.

We denote $\Gamma_{\delta}:=\{x \in \Gamma$; distance $(x, \partial \Gamma)>\delta\}$. If we take $\delta>0$ sufficiently small, by the transversality of $\partial \Delta$ and $\partial \Gamma$ to $\ell$, for any $0 \leq \delta^{\prime} \leq \delta, h^{-1}(\partial \Delta)$ and $h^{-1}\left(\partial \Gamma_{\delta^{\prime}}\right)$ are given by $t=\varphi(x, y)$ and $t=\psi_{\delta^{\prime}}(x, y)$ in $V$ with $\varphi, \psi_{\delta^{\prime}} \in C^{m}(V), \varphi(x, y)>\psi_{\delta^{\prime}}(x, y)$, respectively.

Now, let $\chi(x, y) \in C_{0}^{m}(V), 0 \leq \chi(x, y) \leq 1, \chi(0,0)=1$. We define a family of $C^{m}$ surfaces $\left\{Z_{s}\right\}$ defined in $U$ by

$$
Z_{s}:=\left\{t=s \chi(x, y)\left(\varphi(x, y)-\psi_{s \delta}(x, y)\right)+\psi_{s \delta}(x, y),(x, y) \in V\right\} .
$$

Moreover, we consider the family $\left\{h\left(Z_{S}\right)\right\}$ of $C^{m}$ surfaces defined in $T$ and define a family $\left\{X_{s}\right\}$ of $C^{m}$ surfaces in $\mathbb{R}^{3}$ by

$$
X_{S}= \begin{cases}h\left(Z_{1-s}\right) & \text { in } T \\ \partial \Gamma_{(1-s) \delta} & \text { outside } T .\end{cases}
$$

Since $X_{S}$ is isomorphic to a sphere, $\mathbb{R}^{3} \backslash X_{S}$ has two connected components. We choose the bounded component of $\mathbb{R}^{3} \backslash X_{S}$ as $B_{S}(q)$. Then, this gives the desired strict deformation family of $q$ and $\Gamma$.

\section{References}

1. Alessandrini, G.: Stable determination of conductivity by boundary measurements. Appl. Anal. 27, 153-172 (1988)

2. Ammari, H., Kang, H.: Reconstruction of Small Inhomogeneities from Boundary Measurments. Springer, Berlin Heidelberg New York (2004)

3. Arens, T.: Why linear sampling works. Inverse Probl. 20(1), 163-173 (2004)

4. Cakoni, F., Colton, D.: On the mathematical basis of the linear simpling method. Georgian Math. J. 10, 95-104 (2003)

5. Colton, D., Kirsch, A.: A simple method for solving inverse scattering problems in the resonance region. Inverse Probl. 12, 383-393 (1996)

6. Colton, D., Kress, R.: Inverse Acoustic and Electromagnetic Scattering Theory, 2nd edn. Springer, Berlin Heidelberg New York (1998)

7. Ikehata, M.: Reconstruction of the shape of the inclusion by boundary measurements. Commun. PDE 23, 1459-1474 (1998)

8. Ikehata, M.: Reconstruction of obstacles from boundary measurements. Wave motion 3, 205-223 (1999)

9. Ikehata, M.: A new formulation of the probe method and related problems. Inverse Probl. 21(1), 413-426 (2005)

10. Isakov, V.: Inverse Problems for Partial Differential Equations. Springer Series in Applied Math. Science, vol. 127. Springer, Berlin Heidelberg New York (1998)

11. Kirsch, A.: Characterization of the shape of a scattering obstacle using the spectral data of the far field operator. Inverse probl. 14, 1489-1512 (1998)

12. McLean, W.: Strongly Elliptic Systems and Boundary Integral Equations. Cambridge University press, Cambridge (2000)

13. Luke, D-R., Potthast, R.: The no-response test-a sampling method for inverse acoustic scattering theory. SIAM J. App. Math. 63(4), 1292-1312 (2003)

14. Nakamura, G., Potthast, R., Sini, M.: Unification of the probe and singular sources methods for the inverse boundary value problem by the no-response test. Accepted for publication by Comm. PDE. Available on the page web http://eprints.math.sci.hokudai.ac.jp/ 
15. Nakamura, G., Potthast, R., Sini, M.: A comparative study between some non-iterative methods for the inverse scattering. In: Ammari, H., Kang, H. (eds.) Inverse Problems, Multi-Scale Analysis, and Homogenization. Proceedings of the Workshop in Seoul, 2005. Contemporary Mathematics Volume, American Mathematical Society (To appear, 2006)

16. Nečas, J.: Les méthodes directes en théorie des équations élliptiques. Academia, Prague (1967)

17. Potthast, R.: On the convergence of the no-response test (preprint)

18. Potthast, R.: Point sources and multipoles in inverse scattering theory, vol. 427 of ChapmanHall/CRC, Research Notes in Mathematics. Chapman-Hall/CRC, Boca Raton (2001)

19. Potthast, R., Sylvester, J., Kusiak, S.: A 'range test' for determining scatterers with unknown physical properties. Inverse Probl. 19(3), 533-547 (2003)

20. Potthast, R., Schulz, J.: A multiwave rangetest for obstacle reconstruction with unknown physical properties (preprint)

21. Potthast, R.: Sampling and probe methods - an algorithmical view. Computing 75(2-3), 215-235 (2005)

22. Ramm, A.G.: Scattering by obstacles. D. Reidel Publishing Company, Dordrecht (1986) 